\title{
THE REPRESENTATIONS OF QUANTIZED WALLED BRAUER ALGEBRAS
}

\author{
HEBING RUI AND LINLIANG SONG
}

\begin{abstract}
In this paper, we give a criterion on the semisimplicity of quantized walled Brauer algebras $\mathscr{B}_{r, s}$ and classify its simple modules over an arbitrary field $\kappa$.
\end{abstract}

\section{INTRODUCTION}

Schur-Weyl reciprocities set up close relationship between polynomial representations of general linear groups $G L_{n}$ over $\mathbb{C}$ and representations of symmetric groups $\mathfrak{S}_{r}$ [10. Such results have been generalized in various cases. In [11], Kosuda and Murakami studied mixed Schur-Weyl duality between quantum general linear group $U_{q}\left(\mathfrak{g l}_{n}\right)$ and quantized walled Brauer algebras $\mathscr{B}_{r, s}$ with single parameter over $\mathbb{C}$. Since then, quantized walled Brauer algebras have been studied extensively in [3, 4, 8, 12, etc.

A quantized walled Brauer algebra $\mathscr{B}_{r, s}$ with two parameters was defined by Leduc in [12]. This is a cellular algebra [8] over a commutative ring $R$ containing 1 . In fact, Enyang has shown that any cellular basis of the Hecke algebras associated to symmetric groups can be lifted to a cellular basis of $\mathscr{B}_{r, s}$. In particular, using anti-symmetrizers of Hecke algebras instead of symmetrizers yields a cellular basis of $\mathscr{B}_{r, s}$ in Theorem 3.7 . Our motivation for using this cellular basis is that bases of the corresponding cell modules can be used to classify singular vectors or highest vectors appearing in the mixed tensor product of the natural module and its dual over $U_{q}\left(\mathfrak{g l}_{n}\right)$. Such results can be used to determine decomposition matrices of $\mathscr{B}_{r, s}$. Details will be given in [15].

The aim of this paper is to study the representations of over an arbitrary field $\kappa$ via the representation theory of cellular algebras in [9]. In section 2, we recall the definition of $\mathscr{B}_{r, s}$ and list some of its properties. A cellular basis of $\mathscr{B}_{r, s}$ will be given in section 3 . We use certain idempotents of $\mathscr{B}_{r, s}$ to construct Schur functors in section 4 . We also prove branching rule for cell modules of $\mathscr{B}_{r, s}$. In section 5 , we classify irreducible $\mathscr{B}_{r, s}$-modules over $\kappa$. Finally, we give a criterion on the semisimplicity of $\mathscr{B}_{r, s}$ over $\kappa$. Such a result, which generalizes [11, 6.7], can be considered as a counterpart of [1, Theorem 6.3] for walled Brauer algebras.

\section{The quantized Walled Brauer algebra}

Throughout, we assume that $R$ is the localization of $\mathbb{Z}\left[q, q^{-1}, \rho, \rho^{-1}\right]$ at $q-q^{-1}$, which contains $\delta=\left(\rho-\rho^{-1}\right)\left(q-q^{-1}\right)^{-1}$.

Date: August 14, 2018.

H. Rui was supported partially by NSFC in China and Shanghai Municipal Science and Technology Commission 11XD1402200. 
Definition 2.1. [12] Fix $r, s \in \mathbb{Z}^{>0}$. The quantized walled Brauer algebra $\mathscr{B}_{r, s}$ is the associative $R$-algebra with generators $e_{1}, g_{i}, g_{j}^{*}, 1 \leq i \leq r-1$ and $1 \leq j \leq s-1$ subject to the following relations
a) $\left(g_{i}-q\right)\left(g_{i}+q^{-1}\right)=0,1 \leq i<r$,
h) $\left(g_{i}^{*}-q\right)\left(g_{i}^{*}+q^{-1}\right)=0,1 \leq i<s$,
b) $g_{i} g_{j}=g_{j} g_{i},|i-j|>1$,
i) $g_{i}^{*} g_{j}^{*}=g_{j}^{*} g_{i}^{*},|i-j|>1$,
c) $g_{i} g_{i+1} g_{i}=g_{i+1} g_{i} g_{i+1}, 1 \leq i<r-1$,
j) $g_{i}^{*} g_{i+1}^{*} g_{i}^{*}=g_{i+1}^{*} g_{i}^{*} g_{i+1}^{*}, 1 \leq i<s-1$,
d) $g_{i} e_{1}=e_{1} g_{i}, i \neq 1$,
k) $g_{i}^{*} e_{1}=e_{1} g_{i}^{*}, i \neq 1$,
e) $e_{1} g_{1} e_{1}=\rho e_{1}$
l) $e_{1} g_{1}^{*} e_{1}=\rho e_{1}$,
f) $e_{1}^{2}=\delta e_{1}$,
g) $g_{i} g_{j}^{*}=g_{j}^{*} g_{i}$
m) $e_{1} g_{1}{ }^{-1} g_{1}^{*} e_{1} g_{1}=e_{1} g_{1}^{-1} g_{1}^{*} e_{1} g_{1}^{*}$,
n) $g_{1} e_{1} g_{1}{ }^{-1} g_{1}^{*} e_{1}=g_{1}^{*} e_{1} g_{1}{ }^{-1} g_{1}^{*} e_{1}$.

Remark 2.2. It follows from Definition 2.1 that $\mathscr{B}_{r, s} \cong \mathscr{B}_{s, r}$. When we discuss $\mathscr{B}_{r, s}$, we can assume $r \geq s$ without loss of any generality.

If we allow $s=0$, then $\mathscr{B}_{r, s}$ is the usual Hecke algebra $\mathscr{H}_{r}$ associated to the symmetric group $\mathfrak{S}_{r}$. More explicitly, it is generated by $g_{i}, 1 \leq i \leq r-1$, subject to the defining relations (a)-(c) in Definition 2.1, In general, $\mathscr{B}_{r, s}$ contains two subalgebras which are isomorphic to $\mathscr{H}_{r}$ and $\mathscr{H}_{s}$, respectively.

Lemma 2.3. [8] There is an R-linear anti-involution $\sigma$ on $\mathscr{B}_{r, s}$ which fixes all generators $e_{1}, g_{i}$ and $g_{j}^{*}, 1 \leq i \leq r-1$ and $1 \leq j \leq s-1$.

For convenience, we write $g_{i, j}=g_{i-1} g_{i-2} \cdots g_{j}, i>j, g_{i, i}=1$ and $g_{i, j}=g_{i} g_{i+1} \cdots g_{j-1}$, $i<j$. Similarly, we have the notation $g_{i, j}^{*}$. Given two positive integers $i, j$ with $i \leq r$ and $j \leq s$, let

$$
e_{i, j}=g_{1, i}^{-1} g_{j, 1}^{*} e_{1} g_{1, i}\left(g_{j, 1}^{*}\right)^{-1} \text { and } \bar{e}_{i, j}=g_{1, i}^{-1} g_{j, 1}^{*} e_{1} g_{1, j}^{*} g_{i, 1}^{-1} .
$$

Lemma 2.4. Let $e_{i}=e_{i, i}, i \leq \min \{r, s\}$. Then

a) $e_{i} g_{k}=g_{k} e_{i}, i<k<r$, and $e_{i} g_{l}^{*}=g_{l}^{*} e_{i}, i<l<s$,

b) $e_{i}^{2}=\delta e_{i}, 1 \leq i \leq \min \{r, s\}$,

c) $e_{i} g_{i}^{\varepsilon} e_{i}=e_{i}\left(g_{i}^{*}\right)^{\varepsilon} e_{i}=\rho^{\varepsilon} e_{i}, 1 \leq i<\min \{r, s\}, \varepsilon \in\{1,-1\}$,

d) $e_{i} g_{i}\left(g_{i}^{*}\right)^{-1} e_{i}=e_{i} g_{i}^{*} g_{i}^{-1} e_{i}=e_{i} e_{i+1}=e_{i+1} e_{i}, 1 \leq i<\min \{r, s\}$,

e) $e_{i} e_{i+1} g_{i}=e_{i+1} e_{i} g_{i}^{*}, 1 \leq i<\min \{r, s\}$,

f) $g_{i} e_{i} e_{i+1}=g_{i}^{*} e_{i+1} e_{i}, 1 \leq i<\min \{r, s\}$,

g) $e_{i} e_{j}=e_{j} e_{i}, 1 \leq i, j \leq \min \{r, s\}$.

Proof. (a) follows from Definition 2.1(b),(d),(g),(i),(k). By (2.1),

$$
e_{i}^{2}=g_{i-1}^{-1} g_{i-1}^{*} e_{i-1}^{2} g_{i-1}\left(g_{i-1}^{*}\right)^{-1}=\delta g_{i-1}^{-1} g_{i-1}^{*} e_{i-1} g_{i-1}\left(g_{i-1}^{*}\right)^{-1}=\delta e_{i},
$$

where the second equality follows from induction assumption on $i-1$. This proves (b). By Definition 2.1(g), braid relations, (a), and induction assumption on $i-1$, we have

$$
e_{i} g_{i} e_{i}=g_{i-1}^{-1} g_{i-1}^{*} g_{i}^{-1} e_{i-1} g_{i-1} e_{i-1} g_{i} g_{i-1}\left(g_{i-1}^{*}\right)^{-1}=\rho e_{i} .
$$

One can check $e_{i} g_{i}^{*} e_{i}=\rho e_{i}$, similarly. Finally, the remaining cases of (c) follows from (b) and Definition 2.1(a) or (h). By (a) and braid relations, we have

$$
e_{i} g_{i}^{-1} g_{i}^{*} e_{i} g_{i}=g_{i+1, i-1}^{-1} g_{i-1, i+1}^{*} e_{i-1} g_{i-1}^{-1} g_{i-1}^{*} e_{i-1} g_{i-1} g_{i+1, i-1}\left(g_{i-1, i+1}^{*}\right)^{-1} \text {. }
$$


Using induction assumption on $i-1$ together with (a) and braid relations, we have

$$
e_{i} g_{i}^{-1} g_{i}^{*} e_{i} g_{i}=e_{i} g_{i}^{-1} g_{i}^{*} e_{i} g_{i}^{*}
$$

By similar arguments, we have

$$
g_{i} e_{i} g_{i}^{-1} g_{i}^{*} e_{i}=g_{i}^{*} e_{i} g_{i}^{-1} g_{i}^{*} e_{i}
$$

So, $e_{i} g_{i}^{*} g_{i}^{-1} e_{i}=e_{i} e_{i+1}=e_{i+1} e_{i}$. Further, via these equalities together with (b)-(c) and Definition 2.1(a)(h), we have $e_{i}\left(g_{i}^{*}\right)^{-1} g_{i} e_{i}=e_{i} e_{i+1}$. This proves (d). (e)-(f) follow from (d) and (2.2)-(2.3). Finally, we can assume $i<j$ without loss of any generality. By (a), (d), (e) and (2.1),

$$
\begin{aligned}
e_{i} e_{j} & =e_{i} g_{i, j}^{-1} g_{j, i}^{*} e_{i} g_{i, j}\left(g_{j, i}^{*}\right)^{-1}=g_{i+1, j}^{-1} g_{j, i+1}^{*} e_{i} g_{i}^{-1} g_{i}^{*} e_{i} g_{i, j}\left(g_{j, i}^{*}\right)^{-1} \\
& =g_{i+1, j}^{-1} g_{j, i+1}^{*} e_{i} e_{i+1} g_{i, j}\left(g_{j, i}^{*}\right)^{-1}=g_{i+1, j}^{-1} g_{j, i+1}^{*} e_{i} e_{i+1} g_{i+1, j}\left(g_{j, i+1}^{*}\right)^{-1}=e_{j} e_{i} .
\end{aligned}
$$

This proves $(\mathrm{g})$.

Proposition 2.5. Given $r, s \in \mathbb{Z}^{>0}$, let

$$
c_{r, s}=\sum_{i=1}^{r} \sum_{j=1}^{s} \bar{e}_{i, j}-\rho^{-1} \sum_{i=2}^{r} \sum_{j=1}^{i-1} g_{j, i}^{-1} g_{i, j+1}^{-1}-\rho \sum_{i=2}^{s} \sum_{j=1}^{i-1} g_{i, j}^{*} g_{j+1, i}^{*} .
$$

Then $c_{r, s}$ is central in $\mathscr{B}_{r, s}$.

Proof. If $r+s=2$, then $r=s=1$ and $c_{1,1}=e_{1}$, which is central in $\mathscr{B}_{1,1}$. In the remaining part of the proof, we assume $r+s \geq 3$.

We claim that $e_{1}$ commutes with $c_{r, s}$ by induction on $r+s$. As mentioned in Remark 2.2, we assume $r \geq s$. So, $r \geq 2$ and

$$
c_{r, s}-c_{r-1, s}=\sum_{j=1}^{s} \bar{e}_{r, j}-\rho^{-1} \sum_{j=1}^{r-1} g_{j, r}^{-1} g_{r, j+1}^{-1} .
$$

By induction assumption on $\mathscr{B}_{r-1, s}$, it suffices to prove $e_{1}\left(c_{r, s}-c_{r-1, s}\right)=\left(c_{r, s}-c_{r-1, s}\right) e_{1}$.

In fact, by Lemma 2.4(a),(d),(e), $\bar{e}_{r, j} e_{1}=e_{1} \bar{e}_{r, j}, \forall j>1$. By Definition 2.1(d)-(e) and Lemma 2.4(c),

$$
\bar{e}_{r, 1} e_{1}-e_{1} \bar{e}_{r, 1}=\rho^{-1}\left(g_{1, r}^{-1} g_{r, 2}^{-1} e_{1}-e_{1} g_{1, r}^{-1} g_{r, 2}^{-1}\right) .
$$

Finally, using Definition 2.1(d) yields

$$
e_{1} \sum_{j=2}^{r-1} g_{j, r}^{-1} g_{r, j+1}^{-1}=\sum_{j=2}^{r-1} g_{j, r}^{-1} g_{r, j+1}^{-1} e_{1} .
$$

So, $e_{1}\left(c_{r, s}-c_{r-1, s}\right)=\left(c_{r, s}-c_{r-1, s}\right) e_{1}$, proving our claim.

Now, we prove $c_{r, s} g_{k}=g_{k} c_{r, s}$ for all $k, 1 \leq k \leq r-1$. In fact, by Definition 2.1(b)(c)(i)(j), $\bar{e}_{k, j} g_{i}=g_{i} \bar{e}_{k, j}$ for $k \notin\{i, i+1\}$. Since $\left(\bar{e}_{i+1, j}+\bar{e}_{i, j}\right) g_{i}=g_{i}\left(\bar{e}_{i+1, j}+\bar{e}_{i, j}\right)$, we have

$$
\sum_{i=1}^{r} \sum_{j=1}^{s} \bar{e}_{i, j} g_{k}=g_{k} \sum_{i=1}^{r} \sum_{j=1}^{s} \bar{e}_{i, j}, \text { for all } k, 1 \leq k \leq r-1 .
$$

If we use $g_{k}$ instead of $g_{k}^{-1}, 1 \leq k \leq r-1$ in $x:=\sum_{i=2}^{r} \sum_{j=1}^{i-1} g_{j, i}^{-1} g_{i, j+1}^{-1}$, then $x$ is the summation of the Murphy elements of $\mathscr{H}_{r}$, which is central in $\mathscr{H}_{r}$ (see e.g. [13]). However, 
from Definition 2.1(a)-(c), one can see easily that $\mathscr{H}_{r}$ can be defined via $g_{i}^{-1}$. So $x$ is a central in $\mathscr{H}_{r}$. By Definition [2.1(g), $c_{r, s} g_{k}=g_{k} c_{r, s}, \forall k, 1 \leq k \leq r-1$. Finally, one can check $c_{r, s} g_{k}^{*}=g_{k}^{*} c_{r, s}$, similarly.

It is known that $\mathfrak{S}_{r}$ is generated by $s_{i}$, the basic transposition $(i, i+1), 1 \leq i \leq r-1$. Let $\mathfrak{S}_{r} \times \mathfrak{S}_{s}$ be the product of $\mathfrak{S}_{r}$ and $\mathfrak{S}_{s}$. We use $s_{i}^{*}$ to denote the basic transposition $(i, i+1)$ in $\mathfrak{S}_{s}$.

For convenience, we write $s_{i, j}=s_{i-1} s_{i-1, j}, i>j, s_{i, i}=1$ and $s_{i, j}=s_{i} s_{i+1, j}, i<j$. Similarly, we have the notation $s_{i, j}^{*}$. The following result gives the explicit description on $\mathscr{D}_{r, s}^{f}$ in $[8]$.

Lemma 2.6. Fix $r, s \in \mathbb{Z}^{>0}$ and $f \in \mathbb{N}$ with $f \leq \min \{r, s\}$. Let $\mathfrak{G}_{f}$ be the subgroup of $\mathfrak{S}_{r} \times \mathfrak{S}_{s}$ generated by $s_{i} s_{i}^{*}, 1 \leq i \leq f-1$. Then $\mathscr{D}_{r, s}^{f}$ is a complete set of right coset representatives for $\mathfrak{S}_{r-f} \times \mathfrak{G}_{f} \times \mathfrak{S}_{s-f}$ in $\mathfrak{S}_{r} \times \mathfrak{S}_{s}$ where

$$
\mathscr{D}_{r, s}^{f}=\left\{s_{f, i_{f}} s_{f, j_{f}}^{*} \cdots s_{1, i_{1}} s_{1, j_{1}}^{*} \mid k \leq j_{k}, 1 \leq i_{1}<i_{2}<\cdots<i_{f} \leq r\right\} .
$$

Proof. We denote by $\tilde{\mathscr{D}}_{r, s}^{f}$ the right-hand side of $(2.5)$, and by $\mathscr{D}_{r, s}^{f}$ a complete set of right coset representatives. Then obviously $\tilde{\mathscr{D}}_{r, s}^{f} \subset \mathscr{D}_{r, s}^{f}$.

In order to verify the inverse inclusion, it suffices to prove that $\left|\tilde{\mathscr{D}}_{r, s}^{f}\right|$, the cardinality of $\tilde{\mathscr{D}}_{r, s}^{f}$, is $\frac{r ! s !}{(r-f) !(s-f) ! f !}=C_{r}^{f} C_{s}^{f} f$ !, which is clearly the cardinality of $\mathscr{D}_{r, s}^{f}$, where $C_{r}^{f}$ is the binomial number. This will be done by induction on $f$ as follows.

If $f=0$, there is nothing to be proven. Assume $f \geq 1$. For any element in (2.5), we have $i_{f} \geq f$. For each fixed $i:=i_{f}$, there are $s-f+1$ choices of $j_{f}$ with $j_{f} \geq f$, and further, conditions for other indices are simply conditions for $\mathscr{D}_{i-1, s}^{f-1}$. So,

$$
\begin{aligned}
\left|\tilde{\mathscr{D}}_{r, s}^{f}\right| & =(s-f+1) \sum_{i=f}^{r}\left|\mathscr{D}_{i-1, s}^{f-1}\right| \\
& =(s-f+1) \sum_{i=f}^{r} C_{i-1}^{f-1} C_{s}^{f-1}(f-1) !=\sum_{i=f}^{r} C_{i-1}^{f-1} C_{s}^{f} f !=C_{r}^{f} C_{s}^{f} f !,
\end{aligned}
$$

where the second equality follows from induction assumption on $f$, and the last follows from the well-known combinatorics formula $C_{r}^{i}=C_{r-1}^{i}+C_{r-1}^{i-1}$.

Lemma 2.7. Fix $r, s, f \in \mathbb{Z}^{>0}$ with $f \leq \min \{r, s\}$. Let $\mathscr{B}_{r, s}(f)$ be the subalgebra of $\mathscr{B}_{r, s}$ generated by $e_{f+1}, g_{i}$ and $g_{j}^{*}, f+1 \leq i<r$ and $f+1 \leq j<s$. Then $\mathscr{B}_{r, s}(f) \cong \mathscr{B}_{r-f, s-f}$.

Proof. The required isomorphism sends $e_{f+1}, g_{f+i}, g_{f+j}^{*}$ to $e_{1}, g_{i}, g_{j}^{*}$, respectively. One can compare the defining relations in Definition 2.1 and the equalities in Lemma 2.4.

We denote $\mathscr{B}_{r, s}(f)$ by $R$ if $r=s=f$.

Lemma 2.8. Given a positive integer $f$ with $f \leq \min \{r, s\}$, we have $\sigma\left(e^{f}\right)=e^{f}$ where $e^{f}=e_{1} e_{2} \cdots e_{f}$ and $\sigma$ is given in Lemma 2.3. 
Proof. By Lemma 2.3, $\sigma\left(e^{f}\right)=e^{f}$ if $f=1$. In general, by induction on $f$, we have $\sigma\left(e^{f}\right)=\sigma\left(e_{f}\right) e^{f-1}$. So, we need to prove $\sigma\left(e_{f}\right) e^{f-1}=e^{f}$. In fact,

$$
\begin{aligned}
\sigma\left(e_{f}\right) e^{f-1} & =\left(g_{1, f}^{*}\right)^{-1} g_{f, 1} e_{1} g_{1, f}^{*} g_{f, 1}^{-1} e_{1} \cdots e_{f-1} \\
& =\left(g_{1, f}^{*}\right)^{-1} g_{f, 1} e_{1} g_{1}^{*} g_{1}^{-1} e_{1} g_{2, f}^{*} g_{f, 2}^{-1} e_{2} \cdots e_{f-1} \\
& =\left(g_{1, f}^{*}\right)^{-1} g_{f, 1} e_{1} e_{2} g_{2, f}^{*} g_{f, 2}^{-1} e_{2} \cdots e_{f-1} \\
& =\left(g_{1, f}^{*}\right)^{-1} g_{f, 1} e_{1} e_{2} \cdots e_{f}=e^{f} .
\end{aligned}
$$

We remark that the second, third and forth equalities follow from Lemma 2.4(a) and (d), and the last equality follows from Lemma 2.4(f)-(g).

We define

$$
g_{d}=g_{f, i_{f}} g_{f, j_{f}}^{*} \cdots g_{1, i_{1}} g_{1, j_{1}}^{*} .
$$

for each $d \in \mathscr{D}_{r, s}^{f}$ if $d=s_{f, i_{f}} s_{f, j_{f}}^{*} \cdots s_{1, i_{1}} s_{1, j_{1}}^{*}$ with $j_{k} \geq k$ and $i_{\ell}<i_{\ell+1}$.

The following result is motivated by Yu's work on cyclotomic Birman-Murakami-Wenzl algebras in [16].

Proposition 2.9. Fix $r, s, f \in \mathbb{Z}^{>0}$ with $f \leq \min \{r, s\}$. Let $N_{f}$ be the left $\mathscr{B}_{r, s}(f)$-module generated by $\bar{V}_{r, s}^{f}=\left\{e^{f} g_{d} \mid d \in \mathscr{D}_{r, s}^{f}\right\}$, where $g_{d}$ 's are given in (2.6). Then $N_{f}$ is a right $\mathscr{B}_{r, s}$-module.

Proof. We claim $M_{f}$ is a right $\mathscr{B}_{r, s}$-module, where $M_{f}$ is the left $\mathscr{B}_{r, s}(f)$-module generated by $V_{r, s}^{f}=\left\{e^{f} g_{f, i_{f}} g_{f, j_{f}}^{*} \cdots g_{1, i_{1}} g_{1, j_{1}}^{*} \mid i_{k}, j_{k} \geq k, 1 \leq k \leq f\right\}$.

First, we assume $f=1$. By Definition 2.1(h), and Lemma 2.4(a), (c)-(d), we have

$$
e_{1} g_{1, i_{1}} g_{1, j_{1}}^{*} e_{1}=\left(e_{2} e_{1}+\rho\left(q-q^{-1}\right) e_{1}\right) g_{2, i_{1}} g_{2, j_{1}}^{*} \text { for } i_{1}>1 \text { and } j_{1}>1 \text {. }
$$

Also, we have $e_{1} g_{1, i_{1}} e_{1}=\rho e_{1} g_{2, i_{1}}$ and $e_{1} g_{1, j_{1}}^{*} e_{1}=\rho e_{1} g_{2, j_{1}}^{*}$. In any case, $M_{1}$ is stable under the action of $e_{1}$. By Definition 2.1, it is easy to verify that $M_{1}$ is stable under the actions of $g_{i}$ 's and $g_{j}^{*}$ 's. So, $M_{1}$ is a right $\mathscr{B}_{r, s}$-module, proving our result for $f=1$. Using the result for $f=1$ repeatedly yields the result for general $f$.

By definition, $N_{f} \subseteq M_{f}$. So, our result follows if $M_{f} \subseteq N_{f}$. We prove it by induction on $f$. The case $f=1$ is trivial since $M_{1}=N_{1}$. In general, by induction assumptions on both $f-1$ and $f=1$, we have

$$
\mathscr{B}_{r, s}(f) V_{r, s}^{f} \subseteq \sum_{i_{f}, j_{f} \geq f} \mathscr{B}_{r, s}(f) e_{f} g_{f, i_{f}} g_{f, j_{f}}^{*} \bar{V}_{r, s}^{f-1}
$$

So, $M_{f} \subseteq N_{f}$ if $e_{f} g_{f, i_{f}} g_{f, j_{f}}^{*} e^{f-1} g_{d} \in N_{f}$, for any $e^{f-1} g_{d} \in \bar{V}_{r, s}^{f-1}$.

Write $g_{d}=g_{f-1, i_{f-1}} g_{f-1, j_{f-1}}^{*} \cdots g_{1, i_{1}} g_{1, j_{1}}^{*}$ with $i_{1}<\cdots<i_{f-1}$. If $i_{f}>i_{f-1}$, there is nothing to be proved. So, we assume $i_{f} \leq i_{f-1}$. By Lemma 2.4(e) and Definition 2.1, we have

$$
e_{f} g_{f, i_{f}} g_{f, j_{f}}^{*} e_{f-1} g_{f-1, i_{f-1}} g_{f-1, j_{f-1}}^{*}=e_{f} e_{f-1} g_{f, i_{f-1}} g_{f-1, i_{f}-1} g_{f-1, j_{f}}^{*} g_{f-1, j_{f-1}}^{*},
$$

and

$$
g_{f-1, j_{f}}^{*} g_{f-1, j_{f-1}}^{*}= \begin{cases}g_{f, j_{f-1}}^{*} g_{f-1, j_{f}-1}^{*}+\left(q-q^{-1}\right) g_{f, j_{f}}^{*} g_{f-1, j_{f-1}}^{*}, & \text { if } j_{f-1} \geq j_{f}, \\ g_{f, j_{f-1}+1}^{*} g_{f-1, j_{f}}^{*}, & \text { if } j_{f-1}<j_{f} .\end{cases}
$$


Applying the previous arguments repeatedly yields $e_{f} g_{f, i_{f}} g_{f, j_{f}}^{*} e^{f-1} g_{d} \in \bar{V}_{r, s}^{f}$.

The following result can be considered as the left version of Proposition 2.9,

Corollary 2.10. Fix $r, s, f \in \mathbb{Z}^{>0}$ with $f \leq \min \{r, s\}$. Let $N_{f}$ be the right $\mathscr{B}_{r, s}(f)$-module generated by $\left\{\sigma\left(g_{d}\right) e^{f} \mid d \in \mathscr{D}_{r, s}^{f}\right\}$. Then $N_{f}$ is a left $\mathscr{B}_{r, s}$-module.

Lemma 2.11. Let $I$ be the two-sided ideal of $\mathscr{B}_{r, s}(f)$ generated by $e_{f+1}$. Then $\mathscr{B}_{r, s}(f) / I \cong$ $\mathscr{H}_{r-f} \otimes \mathscr{H}_{s-f}$.

Proof. Straightforward verification.

\section{A cellular basis of $\mathscr{B}_{r, s}$}

The aim of this section is to give a cellular basis of $\mathscr{B}_{r, s}$ in Theorem 3.7. We remark that Enyang [8] has shown that arbitrary cellular bases for Hecke algebras associated to symmetric groups can be lifted to cellular bases of the quantized walled Brauer algebras. In this sense, the cellular basis of $\mathscr{B}_{r, s}$ given in Theorem 3.7 can be obtained from [8, Theorem 6.13].

Definition 3.1. 9] Let $A$ be an $R$-algebra, where $R$ is a commutative ring containing the multiplicative identity 1 . Fix a partially ordered set $\Lambda=(\Lambda, \unrhd)$ and for each $\lambda \in \Lambda$ let $T(\lambda)$ be a finite set. Finally, fix $C_{\mathfrak{s t}} \in A$ for all $\lambda \in \Lambda$ and $\mathfrak{s}, \mathfrak{t} \in T(\lambda)$.

Then the triple $(\Lambda, T, C)$ is a cell datum for $A$ if:

a) $\left\{C_{\mathfrak{s t}} \mid \lambda \in \Lambda\right.$ and $\left.\mathfrak{s}, \mathfrak{t} \in T(\lambda)\right\}$ is an $R$-basis for $A$;

$b)$ the $R$-linear map $*: A \longrightarrow A$ determined by $\left(C_{\mathfrak{s t}}\right)^{*}=C_{\mathfrak{t} \mathfrak{s}}$, for all $\lambda \in \Lambda$ and all $\mathfrak{s}, \mathfrak{t} \in T(\lambda)$ is an anti-involution of $A$;

c) for all $\lambda \in \Lambda, \mathfrak{s} \in T(\lambda)$ and $a \in A$ there exist scalars $r_{\mathfrak{t u}}(a) \in R$ such that

$$
C_{\mathfrak{s t}} a=\sum_{\mathfrak{u} \in T(\lambda)} r_{\mathfrak{t u}}(a) C_{\mathfrak{s u}} \quad\left(\bmod A^{\triangleright \lambda}\right),
$$

where $A^{\triangleright \lambda}=R$-span $\left\{C_{\mathfrak{u v}} \mid \mu \triangleright \lambda\right.$ and $\left.\mathfrak{u}, \mathfrak{v} \in T(\mu)\right\}$. Furthermore, each scalar $r_{\mathfrak{t u}}(a)$ is independent of $\mathfrak{s}$.

An algebra $A$ is a cellular algebra if it has a cell datum. We call $\left\{C_{\mathfrak{s t}} \mid \mathfrak{s}, \mathfrak{t} \in T(\lambda), \lambda \in \Lambda\right\}$ a cellular basis of $A$.

Unless otherwise stated, we always consider right $A$-modules. Via anti-involution in Definition 13.1, all right $A$-modules can be considered as left modules. For each $\lambda \in \Lambda$ fix $\mathfrak{t} \in T(\lambda)$ and let $C_{\mathfrak{s}}=C_{\mathfrak{t} \mathfrak{s}}+A^{\triangleright \lambda}$. The right cell module $C(\lambda)$ of $A$ with respect to $\lambda \in \Lambda$ can be considered as the free $R$-modules with basis $\left\{C_{\mathfrak{s}} \mid \mathfrak{s} \in T(\lambda)\right\}$. Further, for any $a \in A$,

$$
C_{\mathfrak{s}} \cdot a=\sum_{\mathfrak{u} \in T(\lambda)} r_{\mathfrak{s u}}(a) C_{\mathfrak{u}}
$$

where the scalars $r_{\mathfrak{s u}}(a)$ are determined by Definition 3.1(c). Similarly, we have the left cell modules of $A$.

Before we construct a cellular basis of $\mathscr{B}_{r, s}$, we need the Murphy basis for $\mathscr{H}_{n}$, which is a cellular basis in the sense of [9]. First, we recall some combinatorics. 
A composition $\lambda$ of $n$ with at most $d$ parts is a sequence of non-negative integers $\lambda=$ $\left(\lambda_{1}, \lambda_{2}, \ldots, \lambda_{d}\right)$ such that $|\lambda|:=\sum_{i=1}^{d} \lambda_{i}=n$. If $\lambda_{i} \geq \lambda_{i+1}, 1 \leq i \leq d-1$, then $\lambda$ is called a partition of $n$ with at most $d$ parts. Let $\Lambda(d, n)\left(\operatorname{resp} . \Lambda^{+}(d, n)\right)$ be the set of all compositions (resp. partitions) of $n$ with at most $d$ parts. We also use $\Lambda^{+}(n)$ to denote the set of all partitions of $n$. It is known that $\Lambda^{+}(d, n)$ is the poset with dominance order $\unlhd$ as the partial order on it. More explicitly, $\lambda \unlhd \mu$ for $\lambda, \mu \in \Lambda^{+}(d, n)$ if $\sum_{j=1}^{i} \lambda_{j} \leq \sum_{j=1}^{i} \mu_{j}$ for all possible $i \leq d$. Write $\lambda \triangleleft \mu$ if $\lambda \unlhd \mu$ and $\lambda \neq \mu$.

Let $\lambda=\left(\lambda_{1}, \lambda_{2} \ldots\right) \in \Lambda^{+}(n)$. The Young diagram $[\lambda]$ is a collection of boxes (or nodes) arranged in left-justified rows with $\lambda_{i}$ boxes in the $i$-th row of $[\lambda]$. We use $(i, j)$ to denote the box $p$ if $p$ is in $i$-th row and $j$-th column. A box $\left(i, \lambda_{i}\right)$ (resp., $\left.\left(i, \lambda_{i}+1\right)\right)$ is called a removable (resp., addable) node of $\lambda$ (or $[\lambda]$ ) if $\lambda_{i}-1 \geq \lambda_{i+1}$ (resp. $\lambda_{i-1} \geq \lambda_{i}+1$ ). Let $\mathscr{R}(\lambda)$ (resp., $\mathscr{A}(\lambda)$ ) be the set of all removable (resp., addable ) boxes of $\lambda$.

A $\lambda$-tableau $\mathfrak{s}$ is obtained by inserting $i, 1 \leq i \leq n$ into $[\lambda]$ without repetition. A $\lambda$ tableau $\mathfrak{s}$ is said to be standard if the entries in $\mathfrak{s}$ are increasing both from left to right in each row and from top to bottom in each column. Let $\mathscr{T}^{\text {std }}(\lambda)$ be the set of all standard $\lambda$-tableaux.

The symmetric group $\mathfrak{S}_{n}$ acts on a $\lambda$-tableau $\mathfrak{s}$ by permuting its entries. Let $\mathfrak{t}^{\lambda}$ (resp. $\mathfrak{t}_{\lambda}$ ) be the $\lambda$-tableau obtained from the Young diagram $[\lambda]$ by adding $1,2, \cdots, n$ from left to right along the rows (resp. from top to bottom along the columns). For example, if $\lambda=(4,3,1)$, then

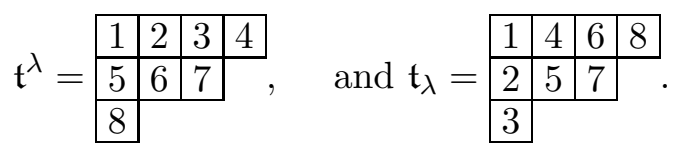

We write $w=d(\mathfrak{s})$ if $\mathfrak{t}^{\lambda} w=\mathfrak{s}$. Then $d(\mathfrak{s})$ is uniquely determined by $\mathfrak{s}$.

Let $\mathcal{Z}=\mathbb{Z}\left[q, q^{-1}\right]$. It is known that $\left\{g_{w} \mid w \in \mathfrak{S}_{n}\right\}$ is a $\mathcal{Z}$-basis of $\mathscr{H}_{n}$, where $g_{w}=$ $g_{i_{1}} \cdots g_{i_{k}}$ if $w=s_{i_{1}} \cdots s_{i_{k}}$ with minimal $k$ which is called the length of $w$. Such an expression is called a reduced expression of $w$. Further, it is well known that $g_{w}$ is independent of a reduced expression of $w$.

Given a $\lambda \in \Lambda^{+}(n)$, let $\mathfrak{S}_{\lambda}$ be the row stabilizer of $\mathfrak{t}^{\lambda}$. Then $\mathfrak{S}_{\lambda}$ is the Young subgroup of $\mathfrak{S}_{n}$ with respect to $\lambda$. Let

$$
\mathfrak{m}_{\lambda}=\sum_{w \in \mathfrak{S}_{\lambda}} q^{\ell(w)} g_{w}, \text { and } \mathfrak{n}_{\lambda}=\sum_{w \in \mathfrak{S}_{\lambda}}(-q)^{-\ell(w)} g_{w}
$$

where $\ell(w)$ is the length of $w$. It is well known that

$$
\mathfrak{m}_{\lambda} g_{i}=q \mathfrak{m}_{\lambda}, \quad \text { and } \quad \mathfrak{n}_{\lambda} g_{i}=-q^{-1} \mathfrak{n}_{\lambda}, \forall s_{i} \in \mathfrak{S}_{\lambda}
$$

For any $\lambda \in \Lambda^{+}(n)$, the classical Specht module $S_{\lambda}$ is $\mathfrak{m}_{\lambda} g_{d\left(\mathfrak{t}_{\lambda}\right)} \mathfrak{n}_{\lambda^{\prime}} \mathscr{H}_{n}$ where $\lambda^{\prime}$ is the conjugate of $\lambda$. The following result is well known.

Proposition 3.2. [5] Suppose $\lambda \in \Lambda^{+}(n)$. Then $\left\{\mathfrak{m}_{\lambda} g_{d\left(\mathfrak{t}_{\lambda}\right)} \mathfrak{n}_{\lambda^{\prime}} g_{d(\mathfrak{t})} \mid \mathfrak{t} \in \mathscr{T}^{\text {std }}\left(\lambda^{\prime}\right)\right\}$ is a $\mathcal{Z}$-basis of $S_{\lambda}$.

In [14, Murphy constructed a $\mathcal{Z}$-basis of $\mathscr{H}_{n}$, called Murphy basis. It is a cellular basis of $\mathscr{H}_{n}$ over $\mathcal{Z}$. In the current paper, we use $\mathfrak{n}_{\lambda}$ instead of $\mathfrak{m}_{\lambda}$ in his construction. The following result follows from Murphy's work in [14]. 
Theorem 3.3. [14] Let $\mathscr{H}_{n}$ be defined over $\mathcal{Z}$. Then $\left\{\mathfrak{n}_{\mathfrak{s t}} \mid \mathfrak{s}, \mathfrak{t} \in \mathscr{T}^{\text {std }}(\lambda), \lambda \in \Lambda^{+}(n)\right\}$ is a cellular basis of $\mathscr{H}_{n}$ where $\mathfrak{n}_{\mathfrak{s t}}=g_{d(\mathfrak{s})-1} \mathfrak{n}_{\lambda} g_{d(\mathfrak{t})}$.

For each $\lambda \in \Lambda^{+}(n)$, let $C(\lambda)$ be the cell module of $\mathscr{H}_{n}$ with respect to this cellular basis. The following result is well known.

Proposition 3.4. For each $\lambda \in \Lambda^{+}(n), S_{\lambda} \cong C\left(\lambda^{\prime}\right)$ where $\lambda^{\prime}$ is the conjugate of $\lambda$.

We begin to construct a cellular basis of $\mathscr{B}_{r, s}$. Fix $r, s \in \mathbb{Z}^{>0}$. Let

$$
\Lambda_{r, s}=\left\{(f, \lambda) \mid \lambda \in \Lambda_{r, s}^{f}, 0 \leq f \leq \min \{r, s\}\right\},
$$

where $\Lambda_{r, s}^{f}=\Lambda^{+}(r-f) \times \Lambda^{+}(s-f)$. So, each $\lambda \in \Lambda_{r, s}^{f}$ is of form $\left(\lambda^{(1)}, \lambda^{(2)}\right)$. We say that $(f, \lambda) \unrhd(\ell, \mu)$ if either $f>\ell$ or $f=\ell$ and $\lambda \unrhd \mu$ in the sense $\lambda^{(i)} \unrhd \mu^{(i)}, i=1,2$. We write $(f, \lambda) \triangleright(\ell, \mu)$ if $(f, \lambda) \unrhd(\ell, \mu)$ and $(f, \lambda) \neq(\ell, \mu)$. Then $\Lambda_{r, s}$ is a poset.

Given a $\lambda \in \Lambda_{r, s}^{f}$, we define $\mathfrak{t}^{\lambda}=\left(\mathfrak{t}^{\lambda^{(1)}}, \mathfrak{t}^{\lambda^{(2)}}\right)$ where $\mathfrak{t}^{\lambda^{(1)}}$ and $\mathfrak{t}^{\lambda^{(2)}}$ are defined similarly as (3.1). The only difference is that we have to use $f+i$ instead of $i$ in (3.1). Similarly, we have $\mathfrak{t}_{\lambda}$.

Example 3.5. Suppose $(r, s)=(2,7), f=1$ and $\left(\lambda^{(1)}, \lambda^{(2)}\right)=((1),(3,2,1))$. We have

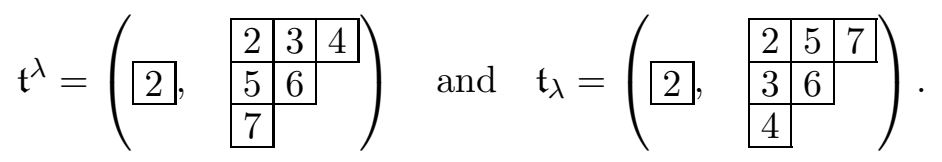

For each $\lambda \in \Lambda_{r, s}^{f}$, let $\mathscr{T}^{\text {std }}\left(\lambda^{(i)}\right)$ be the set of standard $\lambda^{(i)}$-tableaux which are obtained from usual standard tableaux by using $f+j$ instead of $j$. Let $\mathscr{T}^{\text {std }}(\lambda)=\mathscr{T}^{\text {std }}\left(\lambda^{(1)}\right) \times$ $\mathscr{T}^{\text {std }}\left(\lambda^{(2)}\right)$. If $\mathfrak{s}, \mathfrak{t} \in \mathscr{T}^{\text {std }}(\lambda)$ with $\mathfrak{s}=\left(\mathfrak{s}_{1}, \mathfrak{s}_{2}\right)$ and $\mathfrak{t}=\left(\mathfrak{t}_{1}, \mathfrak{t}_{2}\right)$, we define

$$
\mathfrak{n}_{\mathfrak{s t}}=\sigma\left(g_{d\left(\mathfrak{s}_{1}\right)} g_{d\left(\mathfrak{s}_{2}\right)}^{*}\right) \mathfrak{n}_{\lambda^{(1)}} \mathfrak{n}_{\lambda^{(2)}} g_{d\left(\mathfrak{t}_{1}\right)} g_{d\left(\mathfrak{t}_{2}\right)}^{*},
$$

where $\sigma$ is the one given in Lemma 2.3. Let $\mathscr{B}_{r, s}^{f}$ be the two-sided ideal of $\mathscr{B}_{r, s}$ generated by $e^{f}$. Let $\mathscr{B}_{r, s}^{\unrhd(f, \lambda)}$ be the two sided ideal of $\mathscr{B}_{r, s}$ generated by $\mathscr{B}_{r, s}^{f+1}$ and all $e^{f} \mathfrak{n}_{\mathfrak{s t}}$ with $\mathfrak{s}, \mathfrak{t} \in \mathscr{T}^{\text {std }}(\mu)$ and $(f, \mu) \unrhd(f, \lambda)$. Define

$$
\mathscr{B}_{r, s}^{\triangleright(f, \lambda)}=\sum_{(f, \mu) \triangleright(f, \lambda)} \mathscr{B}_{r, s}^{\triangleright(f, \mu) .}
$$

The following result follows from Proposition 2.9 and Lemma 2.11, immediately.

Proposition 3.6. Suppose $(f, \lambda) \in \Lambda_{r, s}$. We have

a) $\Delta^{R}(f, \lambda)$ is a right $\mathscr{B}_{r, s}$-module if $\Delta^{R}(f, \lambda)$ is the R-submodule of $\mathscr{B}_{r, s}^{\unrhd(f, \lambda)} / \mathscr{B}_{r, s}^{\triangleright(f, \lambda)}$ spanned by $\left\{e^{f} \mathfrak{n}_{\mathrm{t}^{\lambda} \mathrm{s}} g_{d}+\mathscr{B}_{r, s}^{\triangleright(f, \lambda)} \mid(\mathfrak{s}, d) \in \mathscr{T}^{\text {std }}(\lambda) \times \mathscr{D}_{r, s}^{f}\right\}$;

b) $\Delta^{L}(f, \lambda)$ is a left $\mathscr{B}_{r, s}$-module if $\Delta^{L}(f, \lambda)$ is the R-submodule of $\mathscr{B}_{r, s}^{\triangleright(f, \lambda)} / \mathscr{B}_{r, s}^{\triangleright(f, \lambda)}$ spanned by $\left\{\sigma\left(g_{d}\right) e^{f} \mathfrak{n}_{\mathrm{st}^{\lambda}}+\mathscr{B}_{r, s}^{\triangleright(f, \lambda)} \mid(\mathfrak{s}, d) \in \mathscr{T}^{s t d}(\lambda) \times \mathscr{D}_{r, s}^{f}\right\}$.

For each $(f, \lambda) \in \Lambda_{r, s}$, we define $I(f, \lambda)=\mathscr{T}^{s t d}(\lambda) \times \mathscr{D}_{r, s}^{f}$. For any $(\mathfrak{s}, e),(\mathfrak{t}, d) \in I(f, \lambda)$, we define

$$
C_{(\mathfrak{s}, e)(\mathfrak{t}, d)}=\sigma\left(g_{e}\right) e^{f} \mathfrak{n}_{\mathfrak{s t}} g_{d} .
$$

As we explained before, the following result can be obtained from [8, Theorem 6.13]. 
Theorem 3.7. Let $\mathscr{B}_{r, s}$ be the quantized wall Brauer algebra over $R$. Then $\mathcal{C}$ is a cellular $R$-basis of $\mathscr{B}_{r, s}$ over the poset $\Lambda_{r, s}$, where

$$
\mathcal{C}=\cup_{(f, \lambda) \in \Lambda_{r, s}}\left\{C_{(\mathfrak{s}, e)(\mathfrak{t}, d)} \mid(\mathfrak{s}, e),(\mathfrak{t}, d) \in I(f, \lambda)\right\} .
$$

The required anti-involution $\sigma$ is the one given in Lemma 2.3.

Proof. Suppose $0 \leq f \leq \min \{r, s\}$. By Proposition 3.6, $\mathscr{B}_{r, s}^{f} / \mathscr{B}_{r, s}^{f+1}$ is spanned by $C_{(\mathfrak{s}, e)(\mathrm{t}, d)}+$ $\mathscr{B}_{r, s}^{f+1}$, for all $(\mathfrak{s}, e),(\mathfrak{t}, d) \in I(f, \lambda)$ and $\lambda \in \Lambda_{r, s}^{f}$. So, $\mathscr{B}_{r, s}$ is spanned by $\mathcal{C}$. Counting the dimension of the walled Brauer algebra $B_{r, s}$ in [1] yields the equality $\# \mathcal{C}=(r+s)$ !. So, $\mathcal{C}$ is $R$-linear independence. Finally, by Proposition 3.6 and Lemma 2.3, $\mathcal{C}$ is a cellular basis in the sense of [9].

For each $(f, \lambda)$, we use $C(f, \lambda)$ to denote the right cell module of $\mathscr{B}_{r, s}$ with respect to the cellular basis in Theorem 3.7. We denote $\lambda^{\prime}$ by $\left(\mu^{(1)}, \mu^{(2)}\right)$ where $\mu^{(i)}$ is the conjugate of $\lambda^{(i)}, i=1,2$.

Proposition 3.8. For each $(f, \lambda) \in \Lambda_{r, s}$, let $\tilde{C}(f, \lambda):=e^{f} \mathfrak{m}_{\lambda^{\prime}} g_{d\left(\mathfrak{t}_{\lambda^{\prime}}\right)} \mathfrak{n}_{\lambda} \mathscr{B}_{r, s}\left(\bmod \mathscr{B}_{r, s}^{f+1}\right)$. As right $\mathscr{B}_{r, s}$-module, $C(f, \lambda) \cong \tilde{C}(f, \lambda)$.

Proof. By Propositions 2.9, $e^{f} \mathfrak{m}_{\lambda^{\prime}} g_{d\left(\mathfrak{t}_{\lambda^{\prime}}\right)} \mathfrak{n}_{\lambda} \mathscr{B}_{r, s}$ is spanned by $e^{f} \mathfrak{m}_{\lambda^{\prime}} g_{d\left(\mathfrak{t}_{\lambda^{\prime}}\right)} \mathfrak{n}_{\lambda} \mathscr{B}_{r, s}(f) g_{d}$ for all $d \in \mathscr{D}_{r, s}^{f}$. By Lemma 2.11, we can use $\mathscr{H}_{r-f} \otimes \mathscr{H}_{s-f}$ instead of $\mathscr{B}_{r, s}(f)$ in $\tilde{C}(f, \lambda)$. Using Proposition 3.2 yields a basis of $e^{f} \mathfrak{m}_{\lambda^{\prime}} g_{d\left(\mathfrak{t}_{\lambda^{\prime}}\right)} \mathfrak{n}_{\lambda} \mathscr{B}_{r, s}\left(\bmod \mathscr{B}_{r, s}^{f+1}\right)$. Now, required isomorphism follows from Proposition 3.4, immediately.

\section{InduCtions And Restrictions}

In this section, unless otherwise stated, we always consider $\mathscr{B}_{r, s}$ over a field $\kappa$. We will describe certain restrictions and inductions of the cell modules of $\mathscr{B}_{r, s}$. This is motivated by Doran, Wales Hanlon's work on Brauer algebras over $\mathbb{C}$ in $[6]$.

Lemma 4.1. Let $\mathscr{B}_{r, s}$ be over $\kappa$. We have

a) $e_{1} \mathscr{B}_{r, s} e_{1}=\mathscr{B}_{r, s}(1) e_{1}$.

b) If $s \geq 2$, then $\tilde{e}_{12} \mathscr{B}_{r, s} \tilde{e}_{1,2}=\mathscr{B}_{r, s}(1) \tilde{e}_{12}$ and $\left(\tilde{e}_{1,2}\right)^{2}=\tilde{e}_{1,2}$ where $\tilde{e}_{1,2}=\rho^{-1} e_{1} g_{1}^{*}$.

c) If $r \geq 2$, then $f_{2,1} \mathscr{B}_{r, s} f_{2,1}=\mathscr{B}_{r, s}(1) f_{21}$ and $\left(f_{2,1}\right)^{2}=f_{2,1}$ where $f_{2,1}=\rho^{-1} e_{1} g_{1}$.

Proof. If $r+s=2$, then $r=s=1$ and $\mathscr{B}_{1,1}(1)=\kappa$. In this case, we have (a) by $e_{1}^{2}=\delta e_{1}$. Suppose $r+s \geq 3$. By Remark 2.2, we can assume $r \geq 2$. Then $e_{1}=\rho^{-1} e_{1} g_{1} e_{1}$. We have $\mathscr{B}_{r, s}(1) e_{1}=e_{1} \mathscr{B}_{r, s}(1) g_{1} e_{1} \subseteq e_{1} \mathscr{B}_{r, s} e_{1}$. By Proposition 2.9 for $f=1$, each element in $e_{1} \mathscr{B}_{r, s} e_{1}$ can be written as a linear combination of elements in $\mathscr{B}_{r, s}(1) e_{1} g_{d} e_{1}$ with $d \in \mathscr{D}_{r, s}^{1}$. Note that $g_{d}=g_{1, i} g_{1, j}^{*}$ for some positive integers $i, j$. By Definition 2.1 (d)(k), we need only to deal with the case $i, j \in\{1,2\}$.

If $\{i, j\} \cap\{1\} \neq \emptyset$, by Definition 2.1 (e) or (f) or (l), $e_{1} g_{d} e_{1} \in \mathscr{B}_{r, s}(1) e_{1}$. Otherwise, by Definition 2.1(a)(l) and Lemma 2.4(d), we have

$$
e_{1} g_{d} e_{1}=e_{1} g_{1} g_{1}^{*} e_{1}=e_{1}\left(g_{1}^{-1}+\left(q-q^{-1}\right)\right) g_{1}^{*} e_{1}=e_{1} e_{2}+\rho\left(q-q^{-1}\right) e_{1} \in \mathscr{B}_{r, s}(1) e_{1} \text {. }
$$

So, $\mathscr{B}_{r, s}(1) e_{1} \supseteq e_{1} \mathscr{B}_{r, s} e_{1}$, and (a) follows. (b)-(c) follow from (a), immediately. 
Corollary 4.2. As algebras over $\kappa$, we have $\mathscr{B}_{r, s}(1) \tilde{e}_{12} \cong \mathscr{B}_{r, s}(1) \cong \mathscr{B}_{r, s}(1) f_{2,1}$.

Proof. Using cellular bases for $\mathscr{B}_{r, s}$ and $\mathscr{B}_{r, s}(1)$ yields bases for $\mathscr{B}_{r, s}(1) \tilde{e}_{12}$ and $\mathscr{B}_{r, s}(1) f_{2,1}$. In particular, we have

$$
\operatorname{dim}_{\kappa} \mathscr{B}_{r, s}(1) \tilde{e}_{12}=\operatorname{dim}_{\kappa} \mathscr{B}_{r, s}(1)=\operatorname{dim}_{\kappa} \mathscr{B}_{r, s}(1) f_{2,1} .
$$

So, the homomorphism from $\mathscr{B}_{r, s}(1)$ to $\mathscr{B}_{r, s}(1) \tilde{e}_{12}\left(\right.$ resp. $\left.\mathscr{B}_{r, s}(1) f_{2,1}\right)$ sending $x$ to $x \tilde{e}_{1,2}$ (resp. $x f_{2,1}$ ) is the required algebra isomorphism.

For any finite dimensional algebra $A$ over $\kappa$, let $A$-mod be the category of left $A$-modules. Since we are considering $\mathscr{B}_{r, s}$, each left $\mathscr{B}_{r, s}$-module can be considered as a right $\mathscr{B}_{r, s}$-module via anti-involution $\sigma$ in Lemma 2.3 .

In the remaining part of this paper, we use $\mathfrak{e}_{r, s}$ to denote either $\tilde{e}_{1,2}$ or $f_{2,1}$ in Lemma 4.5. By Lemma 4.1(b)-(c), Corollary 4.2 and standard arguments in [10, Sect. 6], we have the Schur functor $\mathcal{F}_{r, s}$ and the functor $\mathcal{G}_{r, s}$

$$
\begin{aligned}
\mathcal{F}_{r, s} & : \mathscr{B}_{r, s}-\bmod \longrightarrow \mathscr{B}_{r, s}(1)-\bmod , \\
\mathcal{G}_{r, s}: & \mathscr{B}_{r, s}(1)-\bmod \longrightarrow \mathscr{B}_{r, s}-\bmod ,
\end{aligned}
$$

such that for any left $\mathscr{B}_{r, s}$-module $M$ and any left $\mathscr{B}_{r, s}(1)$-module $N$,

$$
\mathcal{F}_{r, s}(M)=\mathfrak{e}_{r, s} M, \quad \text { and } \quad \mathcal{G}_{r, s}(N)=\mathscr{B}_{r, s} \mathfrak{e}_{r, s} \otimes_{\mathscr{B}_{r, s}(1)} N .
$$

We remark that the right (resp. left) action of $\mathscr{B}_{r, s}(1)$ on $\mathscr{B}_{r, s} \mathfrak{e}_{r, s}\left(\right.$ resp. $\left.\mathfrak{e}_{r, s} M\right)$ is given by

$$
\left(\mathscr{B}_{r, s} \mathfrak{e}_{r, s}\right) \circ h=\mathscr{B}_{r, s} \mathfrak{e}_{r, s} h \mathfrak{e}_{r, s}=\mathscr{B}_{r, s} h \mathfrak{e}_{r, s}
$$

(resp. $h \circ \mathfrak{e}_{r, s} M=\mathfrak{e}_{r, s} h \mathfrak{e}_{r, s} M=h \mathfrak{e}_{r, s} M$ ) for any $h \in \mathscr{B}_{r, s}(1)$.

For the simplification of notation, we use $\mathcal{F}, \mathcal{G}$ and $\mathfrak{e}$ instead of $\mathcal{F}_{r, s}, \mathcal{G}_{r, s}$ and $\mathfrak{e}_{r, s}$. We also use Hom instead of $\operatorname{Hom}_{\mathscr{B}_{r, s}}$ if there is no confusion. By Lemma 2.7, $\mathscr{B}_{r+1, s+1}(1) \cong \mathscr{B}_{r, s}$. By abuse of notation, we will use the same notation to denote the cellular basis, cell modules et al. for $\mathscr{B}_{r+1, s+1}(1)$.

Unlike what we did before, we consider the left cell modules in the remaining part of this section. As we mentioned before, left $\mathscr{B}_{r, s}$-modules can also be considered as right $\mathscr{B}_{r, s}$-modules. We remark that Lemma 4.3 for walled Brauer algebras has been given in [1].

Lemma 4.3. Suppose that $(f, \lambda) \in \Lambda_{r, s}$ and $(\ell, \mu) \in \Lambda_{r+1, s+1}$.

a) $\mathcal{F G}=1$,

b) $\mathcal{F}(C(f, \lambda)) \cong C(f-1, \lambda)$,

c) $\mathcal{G}(C(f, \lambda)) \cong C(f+1, \lambda)$,

d) $\operatorname{Hom}\left(\mathscr{B}_{r+1, s+1} \mathfrak{e}, C(\ell, \mu)\right) \cong \mathfrak{e} C(\ell, \mu)$,

e) $\operatorname{Hom}(\mathcal{G}(C(f, \lambda)), C(\ell, \mu)) \cong \operatorname{Hom}(C(f, \lambda), \mathcal{F}(C(\ell, \mu)))$.

Proof. (a) follows from Lemma 4.1 and Corollary 4.2. We prove (b) under the assumption $f \geq 1$. Otherwise, the result is trivial since $\mathfrak{e} C(0, \lambda)=0$ and $C(-1, \lambda):=0$.

By Lemma 4.1(a), e $C(f, \lambda)$ has a basis $e_{1} \sigma\left(g_{d}\right)\left(e_{2} \cdots e_{f}\right) \mathfrak{n}_{\mathfrak{t t}^{\lambda}}+\mathscr{B}_{r, s}^{\triangleright(f, \lambda)}$ where $d \in \mathscr{D}_{r, s}^{f-1}$ and $\mathfrak{t} \in \mathscr{T}^{s t d}(\lambda)$. In this case, $\mathscr{D}_{r, s}^{f-1}$ consists of elements obtained by using $s_{i}$ instead of $s_{i-1}$ in those of usual $\mathscr{D}_{r, s}^{f-1}$ in Lemma 2.6. So, the required isomorphism between $\mathfrak{e} C(f, \lambda)$ and $C(f-1, \lambda)$ sends $e_{1} \sigma\left(g_{d}\right) e_{2} \cdots e_{f} \mathfrak{n}_{\mathfrak{t t}^{\lambda}}+\mathscr{B}_{r, s}^{\triangleright(f, \lambda)}$ to $\sigma\left(g_{d}\right) e_{2} \cdots e_{f} \mathfrak{n}_{\mathfrak{t t}^{\lambda}}+\mathscr{B}_{r, s}(1)^{\triangleright(f-1, \lambda)}$. 
This proves (b). By general result for rings and idempotents, we have (d). By the adjoint associativity of Hom and tensor functors together with (d), we have (e).

By Corollary 4.2 , the cell module of $\mathscr{B}_{r+1, s+1}(1) \mathfrak{e}$ with respect to $(f, \lambda)$ can be identified with $C(f, \lambda) \mathfrak{e}$, where $C(f, \lambda)$ is the corresponding cell module for $\mathscr{B}_{r+1, s+1}(1)$. Note that $g_{1}$ is invertible. So, $\psi: \mathcal{G}(C(f, \lambda)) \rightarrow C(f+1, \lambda)$ sending $h \mathfrak{e} \otimes e_{2} \cdots e_{f+1} \mathfrak{n}_{\lambda} \mathfrak{e}$ to $h e_{1} e_{2} \cdots e_{f+1} \mathfrak{n}_{\lambda}$ is a homomorphism as left $\mathscr{B}_{r+1, s+1}$-modules. Since $e_{1} e_{2} \cdots e_{f+1} \mathfrak{n}_{\lambda}$ is a generator of $C(f+$ $1, \lambda), \psi$ is an epimorphism. Using Corollary 2.10 for $\mathscr{B}_{r+1, s+1} e_{1}$ and the basis of $C(f, \lambda)$, we have that each element in $\mathcal{G}(C(f, \lambda))$ can be written as a linear combination of elements

$$
\sigma\left(g_{1, i_{1}} g_{1, j_{1}}^{*}\right) \mathfrak{e} \otimes \sigma\left(g_{d}\right) e_{2} \cdots e_{f+1} \mathfrak{n}_{\mathfrak{t t}} \boldsymbol{e} \mathfrak{e}, \quad(\mathfrak{t}, d) \in \mathscr{T}^{s t d}(\lambda) \times \mathscr{D}_{r, s}^{f}, s_{1, i_{1}} s_{1, j_{1}}^{*} \in \mathscr{D}_{r+1, s+1}^{1},
$$

where $\mathscr{D}_{r, s}^{f}$ is obtained from usual $\mathscr{D}_{r, s}^{f}$ by using $s_{i+1}, s_{j+1}^{*}$ instead of $s_{i}$, and $s_{j}^{*}$, for $1 \leq$ $i \leq r-1$ and $1 \leq j \leq s-1$, respectively. Note that $\mathfrak{e} h \mathfrak{e}=h \mathfrak{e}$ for all $h \in \mathscr{B}_{r+1, s+1}(1)$. By (2.7)-(2.8), we can keep those $s_{1, i_{1}} s_{1, j_{1}}^{*} \in \mathscr{D}_{r+1, s+1}^{1}$ such that $s_{1, i_{1}} s_{1, j_{1}}^{*} d \in \mathscr{D}_{r+1, s+1}^{f+1}$. So, $\operatorname{dim}_{\kappa} \mathcal{G}(C(f, \lambda)) \leq \operatorname{dim}_{\kappa} C(f+1, \lambda)$, forcing $\psi$ to be injective. This completes the proof of (c).

Lemma 4.4. Suppose $r \geq 2$ (resp. $s \geq 2$ ). Let $\tilde{\mathscr{B}}_{r-1, s}$ (resp. $\tilde{\mathscr{B}}_{r, s-1}$ ) be the subalgebra of $\mathscr{B}_{r, s}$ generated by $g_{1}^{-1} e_{1} g_{1}, g_{i}, 2 \leq i \leq r-1$ and $g_{j}^{*}, 1 \leq j \leq s-1$ (resp. $\left(g_{1}^{*}\right)^{-1} e_{1} g_{1}^{*}, g_{i}$ and $g_{j}^{*}$ with $1 \leq i \leq r-1$ and $\left.2 \leq j \leq s-1\right)$. Then $\tilde{\mathscr{B}}_{r-1, s} \cong \mathscr{B}_{r-1, s}$ and $\tilde{\mathscr{B}}_{r, s-1} \cong \mathscr{B}_{r, s-1}$.

Proof. It is easy to check the required isomorphism from $\tilde{\mathscr{B}}_{r-1, s}$ (resp. $\tilde{\mathscr{B}}_{r, s-1}$ ) to $\mathscr{B}_{r-1, s}$ (resp. $\mathscr{B}_{r, s-1}$ ) sends $g_{1}^{-1} e_{1} g_{1}$, (resp. $\left.\left(g_{1}^{*}\right)^{-1} e_{1} g_{1}^{*}\right)$ and $g_{i}, g_{j}^{*}$ to $e_{1}$ and $g_{i-1}, g_{j}^{*}$ (resp. $g_{i}, g_{j-1}^{*}$ ).

If $r \geq 2, \mathscr{B}_{r-1, s}$ is a subalgebra of $\mathscr{B}_{r, s}$ with $r-1>0$. So, we consider restriction and induction functors as follows:

$$
\begin{aligned}
\operatorname{Res}_{r, s}^{L}: & \mathscr{B}_{r, s}-\bmod \rightarrow \mathscr{B}_{r-1, s} \text {-mod, } \\
\operatorname{Ind}_{r-1, s}^{L}: & \mathscr{B}_{r-1, s} \text {-mod } \rightarrow \mathscr{B}_{r, s} \text {-mod. }
\end{aligned}
$$

Similarly, we assume that $s \geq 2$, we consider induction and restriction functors as follows.

$$
\begin{aligned}
\operatorname{Res}_{r, s}^{R}: & \mathscr{B}_{r, s} \bmod \rightarrow \mathscr{B}_{r, s-1} \text {-mod, } \\
\operatorname{Ind}_{r, s-1}^{R}: & \mathscr{B}_{r, s-1} \text {-mod } \rightarrow \mathscr{B}_{r, s} \text {-mod. }
\end{aligned}
$$

For the simplification of notations, we will use $\operatorname{Res}^{L}$ instead of $\operatorname{Res}_{r, s}^{L}$, etc.

Lemma 4.5. Let $\mathscr{B}_{r, s}$ be defined over $\kappa$.

a) If $r \geq 2$, then $\mathscr{B}_{r, s} e_{1} g_{1}=\tilde{\mathscr{B}}_{r-1, s} e_{1} g_{1}$.

b) If $s \geq 2$, then $\mathscr{B}_{r, s} e_{1} g_{1}^{*}=\tilde{\mathscr{B}}_{r, s-1} e_{1} g_{1}^{*}$.

Proof. For (a), it suffices to prove $\mathscr{B}_{r, s} e_{1} \subseteq \tilde{\mathscr{B}}_{r-1, s} e_{1}$. By Corollary 2.10, each element in $\mathscr{B}_{r, s} e_{1}$ can be written as a linear combination of elements in $\sigma\left(g_{d}\right) e_{1} \mathscr{B}_{r, s}(1)$ where $d \in \mathscr{D}_{r, s}^{1}$. Note that $d=s_{1, i} s_{1, j}^{*}$ for $i, j \geq 1$. So, we need to verify $g_{1} e_{1} \in \tilde{\mathscr{B}}_{r-1, s} e_{1}$. This is the case since $\rho g_{1} e_{1}=g_{1} e_{1} g_{1} e_{1}=g_{1}^{-1} e_{1} g_{1} e_{1}+\rho\left(q-q^{-1}\right) e_{1}$ and $g_{1}^{-1} e_{1} g_{1} \in \tilde{\mathscr{B}}_{r-1, s}$. Finally, (b) can be proved similarly.

Proposition 4.6. Let $\mathscr{B}_{r, s}$ be over $\kappa$. 
a) If $r \geq 2$, then $\mathscr{B}_{r, s} f_{21} \cong \tilde{\mathscr{B}}_{r-1, s}$ as $\left(\tilde{\mathscr{B}}_{r-1, s}, \mathscr{B}_{r, s}(1) f_{21}\right)$-bimodules.

b) If $s \geq 2$, then $\mathscr{B}_{r, s} \tilde{e}_{12} \cong \tilde{\mathscr{B}}_{r, s-1}$ as $\left(\tilde{\mathscr{B}}_{r-1, s}, \mathscr{B}_{r, s}(1) \tilde{e}_{12}\right)$-bimodules.

Proof. We compute the dimension $\mathscr{B}_{r, s} e_{1}$ via the cellular basis of $\mathscr{B}_{r, s}(1)$, which is obtained from that of $\mathscr{B}_{r-1, s-1}$ by using $g_{i}, g_{j}^{*}$ and $e_{2}$ instead of $g_{i-1}, g_{j-1}^{*}$ and $e_{1}$, respectively.

By Corollary 2.10, each element in $\mathscr{B}_{r, s} e_{1}$ can be written as a linear combination of $\sigma\left(g_{d_{1}} g_{d}\right) e_{1} e_{2} \cdots e_{f} \mathfrak{n}_{\mathfrak{s t}} g_{d_{2}}$ where $\sigma\left(g_{d_{1}}\right) e_{2} \cdots e_{f} \mathfrak{n}_{\mathfrak{s t}} g_{d_{2}}$ ranges over all cellular basis elements of $\mathscr{B}_{r, s}(1)$ and $d \in \mathscr{D}_{r, s}^{1}$. So,

$$
g_{d}=g_{1, i_{1}} g_{1, j_{1}}^{*}, \text { for some } i_{1}, j_{1} \geq 1 .
$$

We remark that the previous $e_{2} \cdots e_{f}$ is 1 if $f=1$.

We claim that each $\sigma\left(g_{d_{1}} g_{d}\right) e^{f}$ can be written as a linear combination of $\sigma\left(g_{a}\right) e^{f}, a \in \mathscr{D}_{r, s}^{f}$. In fact, we prove the similar result for $e^{f} g_{d_{1}} g_{d}$ and use anti-involution to get our claim. We prove it by induction on $f$ as follows.

If $f=1$, there is nothing to be proved. In this case, $d_{1}=1$. If $f=2$, we write $d_{1}=s_{2, i_{2}} s_{2, j_{2}}^{*}$ for some $i_{2}, j_{2} \geq 2$. Since we are assuming (4.1), there is nothing to be proved if $i_{2}>i_{1}$. In this case, $d_{1} d \in \mathscr{D}_{r, s}^{2}$. So, we assume $i_{2} \leq i_{1}$. By Lemma 2.4(e) and Definition 2.1(g), we have

$$
e_{1} e_{2} g_{2, i_{2}} g_{2, j_{2}}^{*} g_{1, i_{1}} g_{1, j_{1}}^{*}=e_{1} e_{2} g_{2, i_{1}} g_{1, i_{2}-1} g_{1}^{*} g_{2, j_{2}}^{*} g_{1, j_{1}}^{*} \text {. }
$$

So, our claim for $f=2$ follows from the special case of (2.8). Using the result for $f=2$ repeatedly yields the result for general $f$.

Now, we count the dimension of $\mathscr{B}_{r, s} e_{1}$. In fact, if we use walled Brauer algebra $B_{r, s}$ (see, e.g. in [1]), the classical limit of $\mathscr{B}_{r, s}$ instead of it, and if we use $s_{i}$ and $s_{j}^{*}$ instead of $g_{i}$ and $g_{j}^{*}$ in a basis of $\mathscr{B}_{r, s} e_{1}$, by our previous arguments, we will get a corresponding basis for $B_{r, s} e_{1}$. So, both $\mathscr{B}_{r, s} e_{1}$ and $B_{r, s} e_{1}$ have the same dimension. By [1, Prop. 2.10], the dimension of $B_{r, s} e_{1}$ is $(r+s-1)$ !. So is $\mathscr{B}_{r, s} e_{1}$. By Lemma 4.5(a), $\phi: \tilde{\mathscr{B}}_{r-1, s} \rightarrow \mathscr{B}_{r, s} \mathfrak{e}$, which sends $h$ to $h \mathfrak{e}, h \in \tilde{\mathscr{B}}_{r-1, s}$ is an epimorphism as left $\tilde{\mathscr{B}}_{r-1, s}$-modules. Comparing the dimensions of $\tilde{\mathscr{B}}_{r-1, s}$ and $\mathscr{B}_{r, s} \mathfrak{e}$ yields the required isomorphism as left $\tilde{\mathscr{B}}_{r-1, s}$-modules.

Note that $\tilde{\mathscr{B}}_{r-1, s} \supset \mathscr{B}_{r, s}(1)$. So, $\tilde{\mathscr{B}}_{r-1, s}$ is a right $\mathscr{B}_{r, s}(1)$-module. By Corollary 4.2, it is a right $\mathscr{B}_{r, s}(1) \mathfrak{e}$-module. More explicitly, if $h \in \tilde{\mathscr{B}}_{r-1, s}$ and $x \mathfrak{e} \in \mathscr{B}_{r, s}(1) \mathfrak{e}$ with $x \in \mathscr{B}_{r, s}(1)$, then the right action of $x \mathfrak{e}$ on $h$ is $h x$. Since $\mathfrak{e} x \mathfrak{e}=x \mathfrak{e}$ for any $x \in \mathscr{B}_{r, s}(1)$, it is routine to check that $\phi$ is a homomorphism as right $\mathscr{B}_{r, s}(1) \mathfrak{e}$-modules. This completes the proof of (a). We remark that (b) can be proved similarly.

We identify $\tilde{\mathscr{B}}_{r-1, s}$ (resp. $\tilde{\mathscr{B}}_{r, s-1}$ ) with $\mathscr{B}_{r-1, s}\left(\right.$ resp. $\left.\mathscr{B}_{r, s-1}\right)$ in the remaining part of this section. The following result follows from Proposition 4.6,

Proposition 4.7. $\operatorname{Res}^{L} \circ \mathcal{G}=\operatorname{Ind} d^{R}$ and $\operatorname{Res}^{R} \circ \mathcal{G}=\operatorname{Ind} d^{L}$.

Given an $(f, \lambda) \in \Lambda_{r, s}$ with $f>0$ and $\lambda=\left(\lambda^{(1)}, \lambda^{(2)}\right)$,

$$
\mathscr{R}\left(\lambda^{(1)}\right)=\left\{p_{i} \mid 1 \leq i \leq a\right\} \text {, and } \mathscr{A}\left(\lambda^{(2)}\right)=\left\{q_{j} \mid 1 \leq j \leq b\right\} .
$$

In the remaining part of this section, we always use $\alpha^{(i)}$ (resp. $\left.\beta^{(j)}\right)$ to denote $\left(\lambda^{(1)} \backslash\right.$ $\left.\left\{p_{i}\right\}, \lambda^{(2)}\right)$ (resp. $\left(\lambda^{(1)}, \lambda^{(2)} \cup\left\{q_{j}\right\}\right)$ ). In other words, $\alpha^{(i)}$ is the bipartition obtained from $\lambda$ 
by removing the node $p_{i}$. Similarly, $\beta^{(j)}$ is the bipartition obtained from $\lambda$ by adding the node $q_{j}$. We arrange $p_{i}$ 's and $q_{j}$ 's such that

$$
\left(f, \alpha^{(1)}\right) \triangleright\left(f, \alpha^{(2)}\right) \triangleright \cdots \triangleright\left(f, \alpha^{(a)}\right) \triangleright\left(f-1, \beta^{(1)}\right) \triangleright \cdots \triangleright\left(f-1, \beta^{(b)}\right) .
$$

Lemma 4.8. Suppose $(f, \lambda) \in \Lambda_{r, s}$ with $\lambda=\left(\lambda^{(1)}, \lambda^{(2)}\right)$. Let $y_{\alpha^{(k)}}=g_{r, a_{k}} \mathfrak{n}_{\lambda} e^{f}+\mathscr{B}_{r, s}^{\triangleright(f, \lambda)} \in$ $C(f, \lambda)$ where $a_{k}=f+\sum_{j=1}^{\ell} \lambda_{j}^{(1)}$ if $p_{k} \in \mathscr{R}\left(\lambda^{(1)}\right)$ with $p_{k}=\left(\ell, \lambda_{\ell}^{(1)}\right)$ for some $\ell$. Then, there is an epimorphism $C\left(f, \alpha^{(k)}\right) \rightarrow N_{k} / N_{k-1}$, where $N_{k}=\sum_{j=1}^{k} \mathscr{B}_{r-1, s} y_{\alpha^{(j)}}, 1 \leq k \leq a$ and $N_{0}=0$.

Proof. Since $y_{\alpha^{(k)}} \in C(f, \lambda)$, we have $N_{k} \subset C(f, \lambda)$. By Corollary 2.10, $\mathscr{B}_{r-1, s} y_{\alpha^{(k)}}$ is spanned by $\sigma\left(g_{d}\right) \mathscr{B}_{r-1, s}(f) y_{\alpha(k)}, d \in \mathscr{D}_{r-1, s}^{f}$.

On the other hand, we have $h e^{f} \equiv \psi_{f}(h) e^{f}\left(\bmod \mathscr{B}_{r, s}^{f+1}\right)$ for any $h \in \mathscr{B}_{r-1, s}(f)$, where $\psi_{f}: \mathscr{B}_{r-1, s}(f) \rightarrow \mathscr{H}_{r-1-f} \otimes \mathscr{H}_{s-f}$ is the epimorphism with kernel $\left\langle e_{f+1}\right\rangle$, the twosided ideal of $\mathscr{B}_{r-1, s}(f)$ generated by $e_{f+1}$. Using the branching rule for the cell module $C\left(\lambda^{(1)}\right)$ for the Hecke algebra $\mathscr{H}_{r-f}$ (see, e.g. [13]), we have that $N_{k} / N_{k-1}$ is spanned by all $\sigma\left(g_{d}\right) \sigma\left(g_{d(\mathfrak{t})}^{*}\right) \sigma\left(g_{d(\mathfrak{v})}\right) y_{\alpha}(k)+N_{k-1}$ where $\mathfrak{v} \in \mathscr{T}^{s t d}\left(\lambda^{(1)} \backslash\left\{p_{k}\right\}\right), \mathfrak{t} \in \mathscr{T}^{\text {std }}\left(\lambda^{(2)}\right)$ and $d \in \mathscr{D}_{r-1, s}^{f}=\left\{d \in \mathscr{D}_{r, s}^{f} \mid(r) d=r\right\}$. Note that $y_{\alpha^{(k)}}=\mathfrak{n}_{\alpha^{(k)}} e^{f} h$, where

$$
h=g_{r, a_{k}} \sum_{i=b_{k}+1}^{a_{k}}(-q)^{a_{k}-i} g_{a_{k}, i} \in \mathscr{B}_{r, s},
$$

where $b_{k}=f+\sum_{i=1}^{\ell-1} \lambda_{i}^{(1)}$. So, the required epimorphism sends $\sigma\left(g_{d}\right) \sigma\left(g_{d(\mathfrak{t})}^{*}\right) \sigma\left(g_{d(\mathfrak{v})}\right) \mathfrak{n}_{\alpha(k)} e^{f}$ to $\sigma\left(g_{d}\right) \sigma\left(g_{d(\mathfrak{t})}^{*}\right) \sigma\left(g_{d(\mathfrak{v})}\right) \mathfrak{n}_{\alpha^{(k)}} e^{f} h$.

We need some combinatorial preparations before we prove the result on the branching rule for $\mathscr{B}_{r, s}$. This is motivated by Enyang's work on Birman-Murakami-Wenzl algebras in 7

Recall that a composition $\mu$ of $n$ is a sequence of non-negative integers $\left(\mu_{1}, \mu_{2}, \ldots\right)$ with $\sum_{i} \mu_{i}=n$. Given a partition $\lambda$ and a composition $\mu$ of $n$, a $\lambda$-tableau $\mathbf{S}$ of content (or type) $\mu$ is the tableau obtained from $Y(\lambda)$ by inserting each box with numbers $i, 1 \leq i \leq n$, such that the number $i$ occurring in $S$ is $\mu_{i}$. If the entries in $S$ are weakly increasing in each row and strictly increasing in each column, $\mathrm{S}$ is called a semi-standard $\lambda$-tableau of content $\mu$. Let $\mathbf{T}^{s s}(\lambda, \mu)$ be the set of all semi-standard $\lambda$-tableaux of content $\mu$. If $\mathbf{T}^{s s}(\lambda, \mu) \neq \emptyset$, then $\lambda \unrhd \mu$.

Let $\mathfrak{s}$ be a $\lambda$-tableau and let $\mu$ be a composition. Then $\mu(\mathfrak{s})$ is the $\lambda$-tableau of type $\mu$ which is obtained from $\mathfrak{s}$ by replacing each entry $i$ in $\mathfrak{s}$ by $j$ if $i$ appears in row $j$ of $\mathfrak{t}^{\mu}$.

Suppose $\mathfrak{s} \in \mathscr{T}^{s t d}(\lambda)$ and $\lambda \in \Lambda^{+}(n)$. Let $\mathfrak{s} \downarrow_{i}$ be obtained from $\mathfrak{s}$ by removing all entries which are strictly bigger than $i$. Then $\mathfrak{s} \downarrow_{i}$ is a standard $\mu$-tableau for some partition $\mu \in \Lambda^{+}(i)$. In this case, we use $\mathfrak{s}_{i}$ instead of $\mu$.

The following result, which has already been used in the proof of [7, Coro. 5.4], can be verified, easily.

Lemma 4.9. Assume $\lambda, \mu \in \Lambda^{+}(n)$ with $\mu_{i}=1$ and $i=l(\mu)$. Suppose $\mathbf{S} \in \mathbf{T}^{s s}(\lambda, \mu)$ and $\mathfrak{s} \in \mathscr{T}^{\text {std }}(\lambda)$ such that $\mu(\mathfrak{s})=\mathrm{S}$. Then $\mathfrak{s}_{n-1} \unrhd \nu$ where $\nu$ is obtained from $\mu$ by removing 
the removable node with maximal row index. Further, $\mathfrak{s}_{n-1}=\nu$ if and only if $\lambda$ is obtained from $\nu$ by adding an addable node.

Lemma 4.10. For each $k, 1 \leq k \leq b$, define

$$
z_{\beta}(k)=\sum_{j=d_{k}}^{c_{k}}(-q)^{j-c_{k}} g_{j, c_{k}}^{*}\left(g_{f, c_{k}}^{*}\right)^{-1} g_{r, f} \mathfrak{n}_{\lambda} e^{f}+\mathscr{B}_{r, s}^{\triangleright(f, \lambda)} \in C(f, \lambda),
$$

where $c_{k}=f+\sum_{j=1}^{\ell} \lambda_{j}^{(2)}, d_{k}=f+\sum_{j=1}^{\ell-1} \lambda_{j}^{(2)}+1$ if $\beta^{(k)}=\left(\lambda^{(1)}, \lambda^{(2)} \cup\left\{q_{k}\right\}\right)$ with $q_{k}=$ $\left(\ell, \lambda_{\ell}^{(2)}+1\right) \in \mathscr{A}\left(\lambda^{(2)}\right)$. Then $z_{\beta^{(k)}} \in \mathscr{B}_{r-1, s} z_{\beta^{(b)}}$ for any $k, 1 \leq k \leq b$.

Proof. By definition,

$$
z_{\beta^{(b)}}=g_{r, f}\left(g_{f, s}^{*}\right)^{-1} \mathfrak{n}_{\lambda} e^{f}+\mathscr{B}_{r, s}^{\triangleright(f, \lambda)} .
$$

It is routine to check $z_{\beta^{(k)}}=\sum_{j=d_{k}}^{c_{k}}(-q)^{j-c_{k}} g_{j, c_{k}}^{*} g_{c_{k}, s}^{*} z_{\beta^{(b)}} \in \mathscr{B}_{r-1, s} z_{\beta^{(b)}}$ for any $k, 1 \leq k \leq$ $b$.

Lemma 4.11. Suppose $(f-1, \mu) \in \Lambda_{r-1, s}$ with $\mu=\left(\lambda^{(1)}, \mu^{(2)}\right) \unrhd \beta^{(b)}$ and $\mu \neq \beta^{(i)}$, $1 \leq i \leq b$. Write $\beta^{(b)}=\left(\lambda^{(1)}, \nu\right)$. If $\mathfrak{t} \in \mathscr{T}^{\text {std }}\left(\lambda^{(1)}\right)$ and $\mathfrak{s} \in \nu^{-1}(\mathrm{~S})$ with $\mathbf{S} \in \mathbf{T}^{s s}\left(\mu^{(2)}, \nu\right)$, then $\mathfrak{n}_{\mu^{(2)}} g_{d(\mathfrak{s})}^{*}\left(g_{f, s}^{*}\right)^{-1} g_{r, f} \mathfrak{n}_{\mathfrak{t t}^{\lambda}(1)} e^{f} \in \mathscr{B}_{r, s}^{\triangleright(f, \lambda)}$.

Proof. For any $\mathfrak{s} \in \nu^{-1}(\mathrm{~S})$ let $\mathfrak{u}=\mathfrak{s} \downarrow_{s-1}$. By Lemma 4.9, $\mathfrak{s}_{s-1} \triangleright \lambda^{(2)}$. For the simplification of notation, we denote $\mathfrak{s}_{s-1}$ by $\tau$. We write $d(\mathfrak{s})=s_{b_{k}, s} d(\mathfrak{u})$ where $b_{k}=f-1+\sum_{i=1}^{k} \mu_{i}^{(2)}$ if we assume that $s$ is in the $k$ th row of $\mathfrak{s}$. So,

$$
\mathfrak{n}_{\mu^{(2)}} g_{d(\mathfrak{s})}^{*}\left(g_{f, s}^{*}\right)^{-1}=\sum_{j=b_{k-1}+1}^{b_{k}}(-q)^{j-b_{k}} g_{j, b_{k}}^{*}\left(g_{f, b_{k}}^{*}\right)^{-1} \mathfrak{n}_{\tau} g_{d(\overline{\mathfrak{u}})}^{*},
$$

where $\overline{\mathfrak{u}}$ is obtained by using $i$ instead of $i-1$ in $\mathfrak{u}$ for all possible $i$ 's. Now, the result follows from $\tau \triangleright \lambda^{(2)}$.

Lemma 4.12. For any $b \in \mathscr{B}_{r-1, s} e^{f-1} \cap \mathscr{B}_{r-1, s}^{f}$,

$$
b \mathfrak{n}_{\beta^{(b)}}\left(g_{f, s}^{*}\right)^{-1} g_{r, f} e_{f}+\mathscr{B}_{r, s}^{\triangleright f, \lambda} \in N_{a} .
$$

Proof. By Corollary 2.10, any $b \in \mathscr{B}_{r-1, s} e^{f-1}$ can be written as a linear combination of elements $\sigma\left(g_{d}\right) e^{f-1} \mathscr{B}_{r-1, s}(f-1)$ with $d \in \mathscr{D}_{r-1, s}^{f-1}$. Then we use the cellular basis of $\mathscr{B}_{r-1, s}(f-1)$ to write any $b \in \mathscr{B}_{r-1, s} e^{f-1} \cap \mathscr{B}_{r-1, s}^{f}$ as a linear combination of elements

$$
\sigma\left(g_{d}\right) e^{f-1} \sigma\left(g_{f, i} g_{f, j}^{*}\right) h e_{f} g_{f, i_{1}} g_{f, j_{1}}^{*}=\sigma\left(g_{d}\right) \sigma\left(g_{f, i} g_{f, j}^{*}\right) h e^{f} g_{f, i_{1}} g_{f, j_{1}}^{*}
$$

with $f \leq i, i_{1} \leq r-1, f \leq j, j_{1} \leq s$ and $h \in \mathscr{B}_{r-1, s}(f)$. We denote $b$ by one of elements in (4.5). Note that

$$
\mathfrak{n}_{\beta^{(b)}} g_{r, f}\left(g_{f, s}^{*}\right)^{-1}=g_{r, f}\left(g_{f, s}^{*}\right)^{-1} \mathfrak{n}_{\lambda} .
$$

where $\mathfrak{n}_{\lambda} \in \mathscr{B}_{r, s}(f)$ and $\mathfrak{n}_{\beta^{(b)}} \in \mathscr{B}_{r-1, s}(f-1)$ (see (4.2)). In order to prove (4.4), by (4.5), it suffices to prove

$$
e^{f} g_{f, i_{1}} g_{f, j_{1}}^{*}\left(g_{f, s}^{*}\right)^{-1} g_{r, f} e_{f} \mathfrak{n}_{\lambda}+\mathscr{B}_{r, s}^{\triangleright f, \lambda} \in N_{a} .
$$


We have

$$
e^{f} g_{f, i_{1}} g_{f, j_{1}}^{*}\left(g_{f, s}^{*}\right)^{-1} g_{r, f} e_{f} \mathfrak{n}_{\lambda}= \begin{cases}\left(g_{f+1, s}^{*}\right)^{-1} g_{r, f+1} e^{f+1} g_{f+1, j_{1}+1}^{*} \mathfrak{n}_{\lambda}, & \text { if } j_{1}<s \\ \rho g_{r, f+1} g_{f+1, i_{1}+1} e^{f} \mathfrak{n}_{\lambda}, & \text { if } j_{1}=s\end{cases}
$$

Obviously, if $j_{1}<s$, then $e^{f} g_{f, i_{1}} g_{f, j_{1}}^{*}\left(g_{f, s}^{*}\right)^{-1} g_{r, f} e_{f} \mathfrak{n}_{\lambda}+\mathscr{B}_{r, s}^{\triangleright f, \lambda}=0 \in N_{a}$.

If $j_{1}=s$, we have $g_{r, f+1} g_{f+1, i_{1}+1} n_{\lambda^{(1)}} \in C\left(\lambda^{(1)}\right)$, where $C\left(\lambda^{(1)}\right)$ is the cell module of $\mathscr{H}_{r-f}(f)$ with respect to $\lambda^{(1)}$ in section 3. So, (4.7) follows from branching rule for cell module $C\left(\lambda^{(1)}\right)$ of Hecke algebra $\mathscr{H}_{r-f}$ in [13].

Suppose $\mathfrak{s}$ is a standard tableau with entries in $f+1, f+2, \cdots, r$. In the following, let $\tilde{\mathfrak{s}}$ be the standard tableau obtained from $\mathfrak{s}$ by using $i-1$ instead of $i$ in $\mathfrak{s}$, for all $i$, $f+1 \leq i \leq r$.

Lemma 4.13. For each $k, 1 \leq k \leq b$, let $M_{k}=N_{a}+\sum_{j=1}^{k} \mathscr{B}_{r-1, s} z_{\beta^{(j)}}$, where $z_{\beta^{(j)}}$ is defined in Lemma 4.10. Then $M_{b} / N_{a}$ is spanned by $\left\{\sigma\left(g_{v}\right) \sigma\left(g_{d(t)}^{*}\right) \sigma\left(g_{d(\tilde{\mathfrak{s}})}\right) z_{\beta^{(k)}}+N_{a} \mid(\tilde{s}, \mathfrak{t}) \in\right.$ $\left.\mathscr{T}^{\text {std }}\left(\beta^{(k)}\right), 1 \leq k \leq b, v \in \mathscr{D}_{r-1, s}^{f-1}\right\}$.

Proof. For any $k, 1 \leq k \leq b$, we have

$$
\begin{aligned}
& z_{\beta^{(b)}}=g_{r, f}\left(g_{f, s}^{*}\right)^{-1} \mathfrak{n}_{\lambda} e^{f}+\mathscr{B}_{r, s}^{\triangleright(f, \lambda)}=\mathfrak{n}_{\beta^{(b)}} g_{r, f}\left(g_{f, s}^{*}\right)^{-1} e^{f}+\mathscr{B}_{r, s}^{\triangleright(f, \lambda)}, \\
& z_{\beta^{(k)}}=\sum_{j=d_{k}}^{c_{k}}(-q)^{j-c_{k}} g_{j, c_{k}}^{*} g_{c_{k}, s}^{*} z_{\beta^{(b)}},
\end{aligned}
$$

where $c_{k}$ and $d_{k}$ are defined in Lemma 4.10. So, $M_{b}$ is generated by $z_{\beta^{(b)}}$ and $N_{a}$. Note that

$$
\mathscr{B}_{r-1, s} z_{\beta^{(b)}}=\mathscr{B}_{r-1, s} e^{f-1} \mathfrak{n}_{\beta^{(b)}} g_{r, f}\left(g_{f, s}^{*}\right)^{-1} e_{f}+\mathscr{B}_{r, s}^{\triangleright(f, \lambda)} .
$$

Applying Corollary 2.10 to $\mathscr{B}_{r-1, s} e^{f-1}$, we have that $\mathscr{B}_{r-1, s} z_{\beta^{(b)}}$ is spanned by

$$
\sigma\left(g_{d}\right) e^{f-1} \mathscr{B}_{r-1, s}(f-1) \mathfrak{n}_{\beta^{(b)}} g_{r, f}\left(g_{f, s}^{*}\right)^{-1} e_{f}+\mathscr{B}_{r, s}^{\triangleright(f, \lambda)},
$$

where $d$ ranges over all elements in $\mathscr{D}_{r-1, s}^{f-1}$. For $x \in \mathscr{B}_{r-1, s}(f-1)$, we can assume that $x$ is in either $\mathscr{H}_{r-f} \otimes \mathscr{H}_{s-f+1}$ or $\mathscr{B}_{r-1, s}^{1}(f-1)$, where $\mathscr{B}_{r-1, s}^{1}(f-1)$ is the two-sided ideal of $\mathscr{B}_{r-1, s}(f-1)$ generated by $e_{f}$.

If $x \in \mathscr{B}_{r-1, s}^{1}(f-1)$, then $\sigma\left(g_{d}\right) e^{f-1} x \in \mathscr{B}_{r-1, s} e^{f-1} \cap \mathscr{B}_{r-1, s}^{f}$. By Lemma 4.12,

$$
\sigma\left(g_{d}\right) e^{f-1} x \mathfrak{n}_{\beta^{(b)}} g_{r, f}\left(g_{f, s}^{*}\right)^{-1} e_{f}+\mathscr{B}_{r, s}^{\triangleright(f, \lambda)} \in N_{a} .
$$

Write $\beta^{(i)}=\left(\lambda^{(1)}, \gamma^{(i)}\right)$. If $x \in \mathscr{H}_{r-f} \otimes \mathscr{H}_{s-f+1}$, then $e^{f-1} x \mathfrak{n}_{\beta^{(b)}} g_{r, f}\left(g_{f, s}^{*}\right)^{-1} e_{f}+\mathscr{B}_{r, s}^{\triangleright(f, \lambda)}$ can be written as a linear combination of elements $e^{f-1} \mathfrak{n}_{\tilde{\mathfrak{s}} \mathfrak{\mathfrak { v }}} \mathfrak{n}_{\mathfrak{t u}} g_{r, f}\left(g_{f, s}^{*}\right)^{-1} e_{f}+\mathscr{B}_{r, s}^{\triangleright(f, \lambda)}$ where $\tilde{\mathfrak{s}} \in \mathscr{T}^{s t d}\left(\lambda^{(1)}\right), \tilde{\mathfrak{v}}=\tilde{\mathfrak{t}}^{\lambda^{(1)}}, \mathfrak{t} \in \mathscr{T}^{s t d}\left(\gamma^{(b)}\right), \mathfrak{u} \in\left(\gamma^{(b)}\right)^{-1}(\mathrm{~S})$ with $\mathbf{S} \in \mathbf{T}^{s s}\left(\mu, \gamma^{(b)}\right)$. By Lemma 4.11, we can assume $\mu \in\left\{\gamma^{(i)} \mid 1 \leq i \leq b\right\}$ in (4.2). Further, if $\mu \in\left\{\gamma^{(i)} \mid 1 \leq i \leq b\right\}$, then there is a unique $\mathbf{S} \in \mathbf{T}^{s s}\left(\gamma^{(i)}, \gamma^{(b)}\right)$ such that the type of $T$ is $\lambda^{(2)}$ where $T$ is obtained from $S$ by removing the node containing the unique largest entry. Further, there is a unique 
$\mathfrak{u} \in\left(\gamma^{(b)}\right)^{-1}(S)$ such that $d(\mathfrak{u})=s_{c_{i}, s}^{*}$, where $c_{i}$ is defined in Lemma 4.10. So,

$$
\begin{aligned}
& e^{f-1} \mathfrak{n}_{\tilde{\mathfrak{s}} \mathfrak{\mathfrak { n }}} \mathfrak{n}_{\mathfrak{t u}} g_{r, f}\left(g_{f, s}^{*}\right)^{-1} e_{f} \equiv \mathfrak{n}_{\tilde{\mathfrak{s}} \mathfrak{\mathfrak { v }}} \mathfrak{n}_{\mathfrak{t} \mathfrak{\gamma} \gamma^{(i)}} g_{c_{i}, s}^{*} g_{r, f}\left(g_{f, s}^{*}\right)^{-1} e^{f} \\
& \quad \equiv \sigma\left(g_{d(\tilde{\mathfrak{s}})}\right) \sigma\left(g_{d(\mathfrak{t})}^{*}\right) \mathfrak{n}_{\lambda^{(1)}} \mathfrak{n}_{\gamma^{(i)}}\left(g_{f, c_{i}}^{*}\right)^{-1} g_{r, f} e^{f} \\
& \quad \equiv \sigma\left(g_{d(\tilde{\mathfrak{s}})}\right) \sigma\left(g_{d(\mathfrak{t})}^{*}\right) \sum_{j=d_{i}}^{c_{i}}(-q)^{j-c_{i}} g_{j, c_{i}}^{*}\left(g_{f, c_{i}}^{*}\right)^{-1} g_{r, f} \mathfrak{n}_{\lambda} e^{f}+\mathscr{B}_{r, s}^{\triangleright(f, \lambda)} \\
& \quad=\sigma\left(g_{d(\tilde{\mathfrak{s}})}\right) \sigma\left(g_{d(\mathfrak{t})}^{*}\right) z_{\beta^{(i)}}
\end{aligned}
$$

Therefore, $M_{b} / N_{a}$ is spanned by the elements, as required.

The following result can be proved similarly.

Lemma 4.14. Let $M_{0}=N_{a}$. Then $M_{k} / M_{k-1}$ is spanned by $\left\{\sigma\left(g_{v}\right) \sigma\left(g_{d(t)}^{*}\right) \sigma\left(g_{d(\tilde{\mathfrak{s}})}\right) z_{\beta^{(k)}}+\right.$ $\left.M_{k-1} \mid(\tilde{\mathfrak{s}}, \mathfrak{t}) \in \mathscr{T}^{\text {std }}\left(\beta^{(k)}\right), v \in \mathscr{D}_{r-1, s}^{f-1}\right\}$, for all $k, 1 \leq k \leq b$.

Theorem 4.15. Suppose $(f, \lambda) \in \Lambda_{r, s}$ with $\lambda=\left(\lambda^{(1)}, \lambda^{(2)}\right)$. Let $N_{i}, 1 \leq i \leq a$ be defined in Lemma 4.8. For $1 \leq j \leq b$, Let $N_{a+j}=M_{j}$, where $M_{j}$ is defined in Lemma 4.13. Then

$$
0 \subset N_{1} \subset N_{2} \subset \cdots \subset N_{a} \subset N_{a+1} \subset \cdots \subset N_{a+b}=C(f, \lambda)
$$

is a filtration of $\mathscr{B}_{r-1, s}$-modules such that

$$
N_{i} / N_{i-1} \cong \begin{cases}C\left(f, \alpha^{(i)}\right), & \text { if } 1 \leq i \leq a, \\ C\left(f-1, \beta^{(i-a)}\right), & \text { if } a+1 \leq i \leq a+b .\end{cases}
$$

Proof. We prove our result under the assumption $f>0$. Otherwise, since $C(0, \lambda) \cong S_{\alpha} \otimes S_{\beta}$ where $\alpha$ and $\beta$ are conjugates of $\lambda^{(1)}$ and $\lambda^{(2)}$, respectively, the result follows from the corresponding result for Hecke algebra. See, e.g [13.

We have constructed a filtration of $M_{b}$ such that there is an epimorphism from $C\left(f, \alpha^{(k)}\right)$ (resp. $\left.C\left(f-1, \beta^{(k)}\right)\right)$ to $N_{k} / N_{k-1}\left(\right.$ resp. $\left.M_{k} / M_{k-1}\right)$. We claim $M_{b}=C(f, \lambda)$.

In fact, by definition of $M_{b}$, we have $M_{b} \subseteq C(f, \lambda)$. Note that any element in $C(f, \lambda)$ can be expressed as a linear combination of elements $\sigma\left(g_{d}\right) \sigma\left(g_{d(\mathfrak{s})}\right) \sigma\left(g_{d(\mathfrak{t})}^{*}\right) \mathfrak{n}_{\lambda} e^{f}+\mathscr{B}_{r, s}^{\triangleright(f, \lambda)}$, where $d \in \mathscr{D}_{r, s}^{f}$ and $(\mathfrak{s}, \mathfrak{t}) \in \mathscr{T}^{s t d}(\lambda)$. Further, $d=s_{f, i_{f}} s_{f, j_{f}}^{*} \cdots s_{1, i_{1}} s_{1, j_{1}}^{*}$ with $i_{k}, j_{k} \geq k$ and $i_{f}>i_{f-1}>\cdots>i_{1}$. If $i_{f}=r$, then

$$
\sigma\left(g_{d}\right) \sigma\left(g_{d(\mathfrak{s})}\right) \sigma\left(g_{d(\mathfrak{t})}^{*}\right) \mathfrak{n}_{\lambda} e^{f} \equiv \sigma\left(g_{d_{1}}\right) \sigma\left(g_{d(\tilde{\mathfrak{s}})}\right) \sigma\left(g_{d(\mathfrak{t})}^{*}\right) g_{r, f} \mathfrak{n}_{\lambda} e^{f}+\mathscr{B}_{r, s}^{\triangleright(f, \lambda)} \in \mathscr{B}_{r-1, s} z_{\beta^{(b)}} \subset M_{b}
$$

where $d_{1}=s_{f, j_{f}}^{*} s_{f-1, i_{f-1}} s_{f-1, j_{f-1}}^{*} \cdots s_{1, i_{1}} s_{1, j_{1}}^{*}$. We remark that the inclusion follows from (4.3). If $i_{f}<r$, then

$$
\sigma\left(g_{d}\right) \sigma\left(g_{d(\mathfrak{s})}\right) \sigma\left(g_{d(\mathfrak{t})}^{*}\right) \mathfrak{n}_{\lambda} e^{f}+\mathscr{B}_{r, s}^{\triangleright(f, \lambda)} \in N_{a} \subset M_{b} .
$$

So, $M_{b} \supseteq C(f, \lambda)$ and hence $M_{b}=C(f, \lambda)$. So,

$\operatorname{dim}_{\kappa} C(f, \lambda)=\sum_{i=1}^{a+b} N_{i} / N_{i-1} \leq \sum_{i=1}^{a} \operatorname{dim}_{\kappa} C\left(f, \alpha^{(i)}\right)+\sum_{j=1}^{b} \operatorname{dim}_{\kappa} C\left(f-1, \beta^{(j)}\right)=\operatorname{dim}_{\kappa} C(f, \lambda)$,

where the last equality follows from branching rule for cell modules for walled Brauer algebras in [1, Theorem 3.3]. So, $\operatorname{dim}_{\kappa} C\left(f, \alpha^{(i)}\right)=\operatorname{dim}_{\kappa} N_{i} / N_{i-1}$ and $\operatorname{dim}_{\kappa} C\left(f-1, \beta^{(j)}\right)=$ 
$\operatorname{dim}_{\kappa} M_{j} / M_{j-1}$, forcing $C\left(f, \alpha^{(i)}\right) \cong N_{i} / N_{i-1}$ and $C\left(f-1, \beta^{(j)}\right) \cong M_{j} / M_{j-1}$, for all possible $i$ and $j$.

\section{The IRreducible $\mathscr{B}_{r, s}$-MOdules}

In this section, we classify the irreducible $\mathscr{B}_{r, s}$-modules over an arbitrary field $\kappa$. First, we briefly recall the representation theory of cellular algebras [9]. At moment, we keep the notations in Definition 3.1. So, $A$ is a cellular algebra over a commutative ring $R$ containing 1 with a cellular basis $\left\{C_{\mathfrak{s t}} \mid \mathfrak{s}, \mathfrak{t} \in T(\lambda), \lambda \in \Lambda\right\}$. Unlike what we have done in section 4 , we consider the right $A$-module in this section. As we mentioned before, each left $A$-module can be considered as as a right $A$-module. The motivation for using right $\mathscr{B}_{r, s}$-module is that bases of right cell modules of $\mathscr{B}_{r, s}$ can be used to classify singular vectors in the mixed tensor product of natural module and its dual over $U_{q}\left(\mathfrak{g l}_{n}\right)$. Details will be given in [15].

Recall that each cell module $C(\lambda)$ of $A$ is the free $R$-module with basis $\left\{C_{\mathfrak{s}} \mid \mathfrak{s} \in T(\lambda)\right\}$. In [9], Graham and Lehrer have proved that every irreducible $A$-module arises in a unique way as the simple head of some cell module. More explicitly, each $C(\lambda)$ comes equipped with the invariant form $\phi_{\lambda}$ which is determined by the equation

$$
C_{\mathfrak{s t}} C_{\mathfrak{t}^{\prime} \mathfrak{s}} \equiv \phi_{\lambda}\left(C_{\mathfrak{t}}, C_{\mathfrak{t}^{\prime}}\right) \cdot C_{\mathfrak{s} \mathfrak{s}} \quad\left(\bmod A^{\triangleright \lambda}\right) .
$$

Consequently,

$$
\operatorname{Rad} C(\lambda)=\left\{x \in C(\lambda) \mid \phi_{\lambda}(x, y)=0 \text { for all } y \in C(\lambda)\right\}
$$

is an $A$-submodule of $C(\lambda)$ and $D^{\lambda}=C(\lambda) / \operatorname{Rad} C(\lambda)$ is either zero or absolutely irreducible. Graham and Lehrer [9] have proved the following result in [9].

Theorem 5.1. 9] Let $(A, \Lambda)$ be a cellular algebra over a field $\kappa$.

a) The set $\left\{D^{\lambda} \mid D^{\lambda} \neq 0\right\}$ consists of a complete set of pairwise non-isomorphic irreducible A-modules.

b) Let $G_{\lambda}$ be the Gram matrix with respect to the invariant form $\phi_{\lambda}$ on $C(\lambda)$. Then $A$ is split semisimple if and only if $\prod_{\lambda \in \Lambda} \operatorname{det} G_{\lambda} \neq 0$ in $\kappa$.

We remark that we will use Theorem 5.1 frequently in sections $5-6$. Via Theorem 3.7 . we have the notion of cell modules for $\mathscr{B}_{r, s}$. We use Theorem 5.1 to classify the irreducible $\mathscr{B}_{r, s}$-module over $\kappa$. Let $\phi_{f, \lambda}$ be the corresponding invariant form on $C(f, \lambda),(f, \lambda) \in \Lambda_{r, s}$.

By abuse of notations, we use $\mathscr{H}_{r-f}\left(\right.$ resp. $\left.\mathscr{H}_{s-f}\right)$ to denote the subalgebra of $\mathscr{B}_{r, s}(f)$ in Lemma 2.11, Then $\left\{\mathfrak{n}_{\mathfrak{s t}} \mid \mathfrak{s}, \mathfrak{t} \in \mathscr{T}^{s t d}(\lambda), \lambda \in \Lambda_{r, s}^{f}\right\}$ is a cellular basis of $\mathscr{H}_{r-f} \otimes \mathscr{H}_{s-f}$. Let $\phi_{\lambda}$ be the invariant form on the cell module $C(\lambda)$ of $\mathscr{H}_{r-f} \otimes \mathscr{H}_{s-f}$ with respect to $\lambda \in \Lambda_{r, s}^{f}$. In the following, we denote $\mathscr{H}_{r-f} \otimes \mathscr{H}_{s-f}$ by $\mathbf{H}(f)$ and use $\mathfrak{n}_{\mathfrak{t}}$ to denote $\mathfrak{n}_{\mathfrak{t} \lambda_{\mathfrak{t}}}+\mathbf{H}(f)^{\triangleright \lambda}$

Lemma 5.2. Let $\mathscr{B}_{r, s}$ be defined over $\kappa$. Suppose $(f, \lambda) \in \Lambda_{r, s}$.

a) If either $r \neq s$ or $r=s$ and $f<r$, then $\phi_{f, \lambda} \neq 0 \Leftrightarrow \phi_{\lambda} \neq 0$,

b) If $r=s=f$, then $\phi_{f, 0}=0 \Leftrightarrow \delta=0$.

Proof. If $\phi_{\lambda} \neq 0$, then $\phi_{\lambda}\left(\mathfrak{n}_{\mathfrak{s}}, \mathfrak{n}_{\mathfrak{t}}\right) \neq 0$ for some $\mathfrak{s}, \mathfrak{t} \in \mathscr{T}^{s t d}(\lambda)$. We have $\phi_{f, \lambda} \neq 0$ since

$$
\mathfrak{n}_{\mathfrak{t}^{\lambda} \mathfrak{s}} e^{f} g_{f+1,1}^{*} e^{f} \mathfrak{n}_{\mathfrak{t} \mathfrak{t}^{\lambda}} \equiv \phi_{\lambda}\left(\mathfrak{n}_{\mathfrak{s}}, \mathfrak{n}_{\mathfrak{t}}\right) \rho^{f} e^{f} \mathfrak{n}_{\mathfrak{t}^{\lambda} \mathfrak{t}^{\lambda}} \quad\left(\bmod \mathscr{B}_{r, s}^{\triangleright(f, \lambda)}\right) .
$$


If $\phi_{f, \lambda} \neq 0$, then $\phi_{f, \lambda}\left(e^{f} \mathfrak{n}_{\mathfrak{s}} g_{d}, e^{f} \mathfrak{n}_{\mathfrak{t}} g_{e}\right) \neq 0$ for some $(\mathfrak{s}, d),(\mathfrak{t}, e) \in I(f, \lambda)$. We have $\phi_{\lambda} \neq 0$. Otherwise, $\mathfrak{n}_{\mathfrak{t}^{\lambda} \mathfrak{s}} h \mathfrak{n}_{\mathfrak{t t}^{\lambda}} \equiv 0\left(\bmod \mathbf{H}(f)^{\triangleright \lambda}\right)$, for all $h \in \mathbf{H}(f)$. Since

$$
e^{f} \mathfrak{n}_{\mathfrak{t}^{\lambda} \mathfrak{s}} g_{d} \sigma\left(g_{e}\right) e^{f} \mathfrak{n}_{\mathfrak{t}^{\lambda}} \equiv \mathfrak{n}_{\mathfrak{t}^{\lambda} \mathfrak{s}} h \mathfrak{n}_{\mathfrak{t} \mathfrak{t}^{\lambda}} e^{f} \quad\left(\bmod \mathscr{B}_{r, s}^{f+1}\right),
$$

we have $\phi_{f, \lambda}\left(e^{f} \mathfrak{n}_{\mathfrak{s}} g_{d}, e^{f} \mathfrak{n}_{\mathfrak{t}} g_{e}\right)=0$, a contradiction. This completes the proof of (a).

Suppose $r=s=f$ and $\delta=0$. Using Lemma 4.1(a) repeatedly yields the following inclusion: $e^{f} g_{d} g_{e}^{*} e^{f} \subseteq e^{f-1} e_{f} \mathscr{B}_{f, f}(f-1) e_{f}$. Therefore, we need to verify $e_{f} \mathscr{B}_{f, f}(f-1) e_{f}=$ 0 , which is equivalent to the equality $e_{1} \mathscr{B}_{1,1} e_{1}=0$. In fact, this follows since $\mathscr{B}_{1,1}=\left\{1, e_{1}\right\}$ and $\delta=0$. Conversely, the result follows from the equalities $e^{f} e^{f}=\delta^{f} e^{f}=0$.

Let $e$ be the least positive integer such that $1+q^{2}+\cdots+q^{2(e-1)}=0$ in $\kappa$. If there is no such an $e$, we set $e=\infty$.

Suppose $\lambda=\left(\lambda_{1}, \lambda_{2}, \cdots\right)$ is a partition. Recall that $\lambda$ is $e$-restricted if $\lambda_{i}-\lambda_{i+1}<e$ for all possible $i$. If $\lambda=\left(\lambda^{(1)}, \lambda^{(2)}\right)$, then $\lambda$ is said to be $e$-restricted if both $\lambda^{(1)}$ and $\lambda^{(2)}$ are $e$-restricted. The following result follows from Lemma 5.2 , immediately.

Theorem 5.3. Let $\mathscr{B}_{r, s}$ be the quantized walled Brauer algebra over the field $\kappa$.

a) If either $\delta \neq 0$ or $\delta=0$ and $r \neq s$, then the non-isomorphic irreducible $\mathscr{B}_{r, s}$-modules are indexed by $\{(f, \lambda) \mid 0 \leq f \leq \min \{r, s\}, \lambda$ being e-restricted $\}$.

b) If $\delta=0$ and $r=s$, then the non-isomorphic irreducible $\mathscr{B}_{r, s}$-modules are indexed by $\{(f, \lambda) \mid 0 \leq f<r, \lambda$ being e-restricted $\}$.

Remark 5.4. Enyang [8] classified the irreducible $\mathscr{B}_{r, s}$-modules by using the conditions $D^{f, \lambda} \neq 0$. However, there is no further information about $(f, \lambda)$ in $[8]$.

The following result follows from Theorem 5.3 and [9, 3.10].

Corollary 5.5. Let $\mathscr{B}_{r, s}$ be the quantized walled Brauer algebra over the field $\kappa$. Then $\mathscr{B}_{r, s}$ is quasi-hereditary in the sense of [2] if and only if $e>\max \{r, s\}$ and either $\delta \neq 0$, or $\delta=0$ and $r \neq s$.

\section{A CRITERION ON THE SEMI-SIMPLICITY OF $\mathscr{B}_{r, s}$}

In this section, we give a necessary and sufficient condition for $\mathscr{B}_{r, s}$ being semisimple over an arbitrary field $\kappa$. We start by recalling Kosuda and Murakami's result as follows.

Lemma 6.1. [11, 6.7] Let $\mathscr{B}_{r, s}$ be defined over $\mathbb{C}$ with $\rho=q^{n}$ and $e=\infty$. Then $\mathscr{B}_{r, s}$ is semisimple if $n \geq r+s$.

Let $c_{r, s} \in \mathscr{B}_{r, s}$ be defined in Proposition 2.5. We want to compute the action of $c_{r, s}$ on each cell module of $C(f, \lambda)$ for all $(f, \lambda) \in \Lambda_{r, s}$. We remark that all cell modules in this sections are left cell modules. Via anti-involution $\sigma$ in Lemma 2.3, they can be considered as right modules.

Definition 6.2. Suppose $(f, \lambda) \in \Lambda_{r, s}$ with $\lambda=\left(\lambda^{(1)}, \lambda^{(2)}\right)$.

a) If $p$ is in the $i$ th row and $j$ th column of the Young diagram $\left[\lambda^{(1)}\right]$, we define

$$
c(p)=\frac{1-q^{2 k}}{q-q^{-1}}
$$


where $k=\operatorname{res}(p)=j-i$.

$b)$ If $p$ is in the $i$ th row and $j$ th column of the Young diagram $\left[\lambda^{(2)}\right]$, we define

$$
c(p)=\frac{1-q^{-2 k}}{q^{-1}-q},
$$

where $k=\operatorname{res}(p)=j-i$.

Lemma 6.3. For $(f, \lambda) \in \Lambda_{r, s}$, let $C(f, \lambda)$ be the cell module of $\mathscr{B}_{r, s}$ with respect to the cellular basis in Theorem 3.7. Then $c_{r, s}$ acts on $C(f, \lambda)$ as scalar $f \delta-\rho^{-1} \sum_{p \in\left[\lambda^{(1)}\right]} c(p)-$ $\rho \sum_{p \in\left[\lambda^{(2)}\right]} c(p) \in R$.

Proof. For any $(f, \lambda) \in \Lambda_{r, s}$, by Lemma 6.1, $\operatorname{det} G_{f, \lambda} \neq 0$ under some specialization of $R$. This implies that $\operatorname{det} G_{f, \lambda} \neq 0$ over the field $F$ of the fractions of $R$. So, $\mathscr{B}_{r, s}$ over $F$ is semisimple and $C(f, \lambda)$ is irreducible. Since $c_{r, s}$ is central, it acts on $C(f, \lambda)$ as scalar over $F$. Since both $C(f, \lambda)$ and $c_{r, s}$ are defined over $R, c_{r, s}$ acts on $C(f, \lambda)$ as scalar over $R$, too. So, we need only prove

$$
c_{r, s} y=\left(f \delta-\rho^{-1} \sum_{p \in\left[\lambda^{(1)}\right]} c(p)-\rho \sum_{p \in\left[\lambda^{(2)}\right]} c(p)\right) y
$$

where $y=e^{f} \mathfrak{n}_{\lambda}+\mathscr{B}_{r, s}^{\triangleright(f, \lambda)}$. It is easy to see that $e_{i, i} e^{f}=\delta e^{f}$ for $1 \leq i \leq f$ and $\bar{e}_{i, j} e^{f} \in \mathscr{B}_{r, s}^{f+1}$, if $f<i, j$. Further,

$$
\bar{e}_{i, j} e^{f}= \begin{cases}\rho^{-1} g_{j, i}^{-1} g_{i, j+1}^{-1} e^{f}, & \text { if } 1 \leq j \leq f<i, \\ \rho g_{j, i}^{*} g_{i+1, j}^{*} e^{f}, & \text { if } 1 \leq i \leq f<j, \\ \rho g_{j, i}^{*} g_{i+1, j}^{*} e^{f}, & \text { if } 1 \leq i<j \leq f, \\ \rho^{-1} g_{j, i}^{-1} g_{i, j+1}^{-1} e^{f}, & \text { if } 1 \leq j<i \leq f .\end{cases}
$$

Therefore,

$$
c_{r, s} y=\left\{f \delta-\rho^{-1} \sum_{j=f+2}^{r} \sum_{i=f+1}^{j} g_{w_{i j}}^{-1}-\rho \sum_{j=f+2}^{s} \sum_{i=f+1}^{j} g_{w_{i j}}^{*}\right\} e^{f} \mathfrak{n}_{\lambda},
$$

where $w_{i j}=(i, j)$, the transposition which switches $i$ and $j$. Now, the result follows from the arguments similar to those for Hecke algebras in [13, 3.32].

In the remaining part of this section, unless otherwise stated, we assume that $e>$ $\max \{r, s\}$. Otherwise, $\mathscr{H}_{r} \otimes \mathscr{H}_{s}$ is not semisimple over $\kappa$. So is $\mathscr{B}_{r, s}$.

Let $\lambda$ and $\mu$ be two partitions. We write $\lambda \supseteq \mu$ if $\lambda_{i} \geq \mu_{i}$ for all possible $i$ 's. Let $[\lambda / \mu]$ be the skew Young diagram obtained from $[\lambda]$ by removing nodes in $[\mu]$.

For general cellular algebra $(A, \Lambda)$, we use $\left[C(\lambda): D^{\mu}\right]$ to denote the multiplicity of irreducible $A$-module $D^{\mu}$ in the cell module $C(\lambda)$.

Lemma 6.4. Suppose $(0, \lambda),(1, \mu) \in \Lambda_{r, s}$. If $[C(0, \lambda): C(1, \mu)] \neq 0$, then $\lambda^{(i)} \supset \mu^{(i)}$ and $\left[\lambda^{(i)} / \mu^{(i)}\right]=\left\{p_{i}\right\}$ for some $p_{i} \in \mathscr{R}\left(\lambda^{(i)}\right)$ such that $\rho^{2}=q^{2 k}$ and $k=\operatorname{res}\left(p_{1}\right)+\operatorname{res}\left(p_{2}\right)$.

Proof. We prove our result by induction on $r+s$. The case $r+s=2$ is trivial. In this case, $\lambda=((1),(1))$ and $\mu=(\emptyset, \emptyset)$. In general, we assume $r \geq 2$. Otherwise, we switch the role between $r$ and $s$ in the following arguments. 
Since we are assuming $e>\max \{r, s\}$, we have $C(0, \lambda)=D^{0, \lambda}$ for any $\lambda \in \Lambda_{r, s}^{0}$. We consider the restriction of $C(0, \lambda)$ to $\mathscr{B}_{r-1, s}$. Note that any composition factor of $C(0, \lambda)$ is of form $C\left(0,\left(\lambda^{(1)} \backslash\{p\}, \lambda^{(2)}\right)\right)$ for some $p \in \mathscr{R}\left(\lambda^{(1)}\right)$. By Theorem4.15, there is a $p \in \mathscr{R}\left(\lambda^{(1)}\right)$ such that either

$$
\left[C\left(1,\left(\mu^{(1)} \backslash\{\tilde{p}\}, \mu^{(2)}\right)\right): C\left(0,\left(\lambda^{(1)} \backslash\{p\}, \lambda^{(2)}\right)\right)\right] \neq 0 \text {, for some } \tilde{p} \in \mathscr{R}\left(\mu^{(1)}\right),
$$

or

$$
\left[C\left(0,\left(\mu^{(1)}, \mu^{(2)} \cup\left\{p_{2}\right\}\right)\right): C\left(0,\left(\lambda^{(1)} \backslash\{p\}, \lambda^{(2)}\right)\right)\right] \neq 0 \text {, for some } p_{2} \in \mathscr{A}\left(\mu^{(2)}\right) .
$$

In the first case, by induction assumption, $\lambda^{(1)} \backslash\{p\} \supset \mu^{(1)} \backslash\{\tilde{p}\}$ and $\lambda^{(2)} \supset \mu^{(2)}$. Using Lemma 6.3 yields $c(p)=c(\tilde{p})$. Since we are assuming $e>\max \{r, s\}$, we have $p=\tilde{p}$. So, $\lambda^{(i)} \supset \mu^{(i)}$ and $\left[\lambda^{(i)} / \mu^{(i)}\right]=\left\{p_{i}\right\}$ for some $p_{i}, i=1,2$. In the second case, since $\mathscr{H}_{r-1} \otimes \mathscr{H}_{s}$ is semisimple, we have $\left(\lambda^{(1)} \backslash\{p\}, \lambda^{(2)}\right)=\left(\mu^{(1)}, \mu^{(2)} \cup\left\{p_{2}\right\}\right)$. We still have $\lambda^{(i)} \supset \mu^{(i)}$ and $\left[\lambda^{(i)} / \mu^{(i)}\right]=\left\{p_{i}\right\}$ with $p_{1}=p$. Finally, using Lemma 6.3 yields $\rho^{2}=q^{2 k}$ with $k=\operatorname{res}\left(p_{1}\right)+\operatorname{res}\left(p_{2}\right)$, as required.

Lemma 6.5. Suppose $\lambda=((r-1), \emptyset)$ with $r \geq 2$. We have $\operatorname{det} G_{1, \lambda}=0$ if and only if $\rho^{2} \in\left\{q^{-2}, q^{2 r-2}\right\}$.

Proof. If $\operatorname{det} G_{1, \lambda}=0$, then $\operatorname{Rad} C(1, \lambda) \neq 0$. So, there is an irreducible $\mathscr{B}_{r, 1}$-module, say $D^{\ell, \mu}$ such that $D^{\ell, \mu} \subset \operatorname{Rad} C(1, \lambda)$ and $(\ell, \mu) \triangleleft(1, \lambda)$. Therefore, $\ell \in\{0,1\}$.

We have $\ell=0$. Otherwise, there is a non-trivial homomorphism from $C(1, \mu)$ to $C(1, \lambda)$. Applying the functor $\mathcal{F}$ to both $C(1, \mu)$ and $C(1, \lambda)$ and using Lemma 4.3(b) yields a non-trivial homomorphism from $C(0, \mu)$ to $C(0, \lambda)$, forcing $\lambda=\mu$, a contradiction. When $\ell=0$, by Lemma 6.4, we have $\rho^{2} \in\left\{q^{-2}, q^{2 r-2}\right\}$ if $\operatorname{det} G_{1, \lambda}=0$. Finally, we need to verify $\operatorname{det} G_{1, \lambda}=0$ if $\rho^{2} \in\left\{q^{-2}, q^{2 r-2}\right\}$.

Let $v_{i}=g_{i, 1} \mathfrak{n}_{\lambda} e_{1}+\mathscr{B}_{r, 1}^{\triangleright(1, \lambda)} \in C(1, \lambda)$. Then the Gram matrix $G_{1, \lambda}$ is the $r \times r$ matrix $\left(a_{i j}\right)$ with entry $a_{i, j}=\left\langle v_{i}, v_{j}\right\rangle$. More explicitly, $\operatorname{det} G_{1, \lambda}=0$ if and only if

$$
\left|\begin{array}{ccccc}
\rho^{-1} \delta & 1 & -q^{-1} & \cdots & (-q)^{2-r} \\
1 & \rho^{-1} \delta+q-q^{-1} & (-q)^{-2} & \cdots & (-q)^{1-r} \\
-q^{-1} & (-q)^{-2} & \rho^{-1} \delta+q-q^{-3} & \cdots & (-q)^{-r} \\
\cdot & \cdot & \cdot & \cdots & \cdot \\
\cdot & \cdot & \cdot & \cdots & \cdot \\
(-q)^{2-r} & (-q)^{1-r} & (-q)^{-r} & \cdots & \rho^{-1} \delta+q-q^{3-2 r}
\end{array}\right|=0 .
$$

Let $\operatorname{det}\left(b_{i j}\right)$ be the left hand side of the above equality. We have $b_{2, j}=-q^{-1} b_{1, j}, 1 \leq j \leq r$ if $\rho^{2}=q^{-2}$. When $\rho^{2}=q^{2(r-1)}, \sum_{j=1}^{r}(-q)^{1-j} b_{i, j}=0,1 \leq i \leq r$. In any case, we have $\operatorname{det} G_{1, \lambda}=0$.

By similar arguments, we have the following result.

Lemma 6.6. Suppose $\lambda=\left(\left(1^{r-1}\right), \emptyset\right)$ with $r \geq 2$. Then $\operatorname{det} G_{1, \lambda}=0$ if and only if $\rho^{2} \in\left\{q^{2}, q^{2-2 r}\right\}$.

Lemma 6.7. If $\rho^{2}=q^{2(r+s-2)}$, then there is a $\mathscr{B}_{r, s}$-submodule $M \subset C(1, \mu)$ with $\mu=$ $((r-1),(s-1))$ such that $M \cong C(0, \lambda)$ with $\lambda=((r),(s))$. 
Proof. We consider $\kappa$-space $M$ spanned by $v=\mathfrak{n}_{\lambda} e_{1} \mathfrak{n}_{\mu}+\mathscr{B}_{r, s}^{\triangleright(1, \mu)} \in C(1, \mu)$. Since we are assuming that $e>\max \{r, s\}, v \neq 0$, forcing $M \cong C(0, \lambda)$ as left $\mathscr{H}_{r} \otimes \mathscr{H}_{s}$-modules. It is routine to check that

$$
e_{1} v=\left(\delta-\rho \frac{1-q^{-2(r+s-2)}}{q-q^{-1}}\right) e_{1} \mathfrak{n}_{\mu}+\mathscr{B}_{r, s}^{\triangleright(1, \mu)},
$$

which is zero if $\rho^{2}=q^{2(r+s-2)}$. So, $M \cong C(0, \lambda)$ as $\mathscr{B}_{r, s}$-submodules.

The following result can be proved similarly. The only difference is that we have to use $\mathfrak{m}_{\lambda^{\prime}}$ instead of $\mathfrak{n}_{\lambda}$ in the proof of Lemma 6.7

Lemma 6.8. If $\rho^{2}=q^{-2(r+s-2)}$, then there is a $\mathscr{B}_{r, s}$ submodule $M \subset C(1, \mu)$ with $\mu=$ $\left(\left(1^{r-1}\right),\left(1^{s-1}\right)\right)$ such that $M \cong C(0, \lambda)$ with $\lambda=\left(\left(1^{r}\right),\left(1^{s}\right)\right)$.

Proposition 6.9. Suppose $r, s \in \mathbb{Z}^{>0}$ and $\delta \neq 0$. If $e>\max \{r, s\}$, then $\mathscr{B}_{r, s}$ is semisimple if and only if $\prod_{\lambda} \operatorname{det} G_{1, \lambda} \neq 0$ in $\kappa$ where $\lambda \in \cup_{k=2}^{r+s-1}\left\{((k-1), \emptyset),\left(\left(1^{k-1}\right), \emptyset\right)\right\}$.

Proof. We prove our result by induction on $r+s$ for all possible $r, s \in \mathbb{Z}^{>0}$. We assume $r+s>2$ since $\mathscr{B}_{1,1}$ is semisimple. When $r+s=3$, any cell module of $\mathscr{B}_{r, s}$ is of form either $C(1, \lambda)$ or $C(0, \lambda)$. Note that $(r, s)=\{(2,1),(1,2)\}$. By Lemma 6.5, $\operatorname{det} G_{1, \lambda} \neq 0$ if and only if $\operatorname{det} G_{1, \mu} \neq 0$ where $\lambda=((1),(0))$ and $\mu=((0),(1))$. By Theorem 5.1(b), we have the result. In the remaining part of the proof, we assume that $r+s \geq 4$.

" $\Leftarrow$ " If $\mathscr{B}_{r, s}$ is not semisimple over $\kappa$, by Theorem 5.1 (b), we have $\operatorname{det} G_{f, \lambda}=0$ for some $(f, \lambda) \in \Lambda_{r, s}$. Since we are assuming $e>\max \{r, s\}, \mathscr{H}_{r} \otimes \mathscr{H}_{s}$ is semisimple. Note that any cell module $C(0, \lambda)$ of $\mathscr{B}_{r, s}$ with $(0, \lambda) \in \Lambda_{r, s}$ can be considered as the corresponding cell module for $\mathscr{H}_{r} \otimes \mathscr{H}_{s}$. We have $\operatorname{det} G_{0, \lambda} \neq 0$. Therefore, $f>0$. We prove our result by induction on $r+s$.

We can find a simple module, say $D^{\ell, \mu}$ such that $D^{\ell, \mu} \subseteq \operatorname{Rad} C(f, \lambda)$. So, $(\ell, \mu) \triangleleft(f, \lambda)$. In particular, $\ell \leq f$. It results in a non-zero homomorphism from $C(\ell, \mu)$ to $C(f, \lambda)$. We claim $\ell=0$. Otherwise, applying the functor $\mathcal{F}$ to both $C(\ell, \mu)$ and $C(f, \lambda)$ and using Lemma 4.3 yields a non-zero homomorphism from $C(0, \mu)$ to $C(f-\ell, \lambda)$. So, $\mathscr{B}_{r_{1}, s_{1}}$ is not semisimple where $r_{1}=r-\ell, s_{1}=s-\ell$. This contradicts our induction assumption on $r+s-2 \ell$. Now, we assume $\ell=0$ and $f \geq 1$.

We can assume $r \geq s$. Otherwise, we switch the role between $r$ and $s$ in the following statements. So, $r \geq 2$. We consider $C(0, \mu)$ as $\mathscr{B}_{r-1, s}$-module. In this case, let $C(0, \nu)$ be a composition factor of $C(0, \mu)$ for some $\nu$ with $|\nu|=|\mu|-1$. By Theorem 4.15, $C(0, \nu)$ has to be a composition factor of a cell module, say $C\left(f_{1}, \alpha\right)$ for $\mathscr{B}_{r-1, s}$. Further, $f_{1} \geq f-1 \geq 0$. So, $\mathscr{B}_{r-1, s}$ is not semisimple over $\kappa$ if $f>1$, a contradiction. When $f=1$, since we are assuming that $\delta \neq 0$, which is equivalent to $\rho^{2} \neq 1$, by Lemmas 6.4 6.6, there is a $\lambda \in \cup_{k=2}^{r+s-1}\left\{((k-1), \emptyset),\left(\left(1^{k-1}\right), \emptyset\right)\right\}$, such that $\operatorname{det} G_{1, \lambda}=0$, a contradiction.

$" \Rightarrow "$ Suppose $\operatorname{det} G_{1, \lambda}=0$, for some $\lambda \in\left\{((k-1), \emptyset),\left(\left(1^{k-1}\right), \emptyset\right)\right\}$ in $\kappa$. We claim that $k \in\{r+s-1, r+s-2\}$. Otherwise, by induction on $r+s-3, \mathscr{B}_{r-1, s-1}$ is not semisimple. Therefore, $\operatorname{det} G_{\ell, \mu}=0$ for some $(\ell, \mu) \in \Lambda_{r-1, s-1}$. Applying the functor $\mathcal{G}$ to the cell module $C(\ell, \mu)$ yields $\operatorname{det} G_{\ell+1, \mu}=0$. This contradicts our assumption that $\mathscr{B}_{r, s}$ is semisimple. Therefore, $k \in\{r+s-1, r+s-2\}$. By Lemmas 6.5]6.6, $\rho^{2} \in\left\{q^{ \pm 2}, q^{ \pm 2(r+s-2)}, q^{ \pm 2(r+s-3)}\right\}$. 
In fact, $\rho^{2} \neq q^{ \pm 2}$. Otherwise, $\mathscr{B}_{r-1, s-1}$ is not semisimple. So, $\mathscr{B}_{r, s}$ is not semisimple, either. If $\rho^{2} \neq q^{ \pm 2(r+s-2)}$, by Lemmas 6.7 6.8, $\mathscr{B}_{r, s}$ is not semisimple.

Suppose $\rho^{2}=q^{2(r+s-3)}$. We can assume $r+s>4$. Otherwise, $\rho^{2}=q^{2}$, which has already been discussed. By Remark 2.2, we can assume $r \geq 3$.

By Lemma 6.7. $\operatorname{Hom}(C(0, \lambda), C(1, \mu)) \neq 0$ where $\lambda=((r-1),(s))$ and $\mu=((r-$ $2),(s-1))$. By Theorem 4.15, Hom $\left(C(0, \lambda), \operatorname{Res}^{L} C(1, \nu)\right) \neq 0$ with $\nu=((r-2,1),(s-$ 1)). By Frobenius reciprocity, $\operatorname{Hom}\left(\operatorname{Ind}^{L} C(0, \lambda), C(1, \nu)\right) \neq 0$. By Proposition 4.7, and Theorem 4.15, there is a filtration

$$
0 \subset C(1,(r-1),(s-1)) \subset M \subset \operatorname{Ind}^{L} C(0, \lambda)
$$

such that

$$
M / C(1,(r-1),(s-1)) \cong C(0,(r),(s))
$$

and

$$
\text { Ind }^{L} C(0, \lambda) / M \cong C(0,((r-1,1),(s))) .
$$

By Lemma 6.4 $\operatorname{Hom}(N, C(1, \nu))=0$ if $N=C(0,(r),(s))$. Since we are assuming that $e>\max \{r, s\}, \operatorname{Hom}(N, C(1, \nu))=0$ if $N=C(1,(r-1),(s-1))$. So,

$$
\operatorname{Hom}(C(0,(r-1,1),(s-1)), C(1, \nu)) \neq 0,
$$

forcing $\mathscr{B}_{r, s}$ being non semisimple, a contradiction. So, $\rho^{2} \neq q^{2(r+s-3)}$. Finally, we remark that we can prove $\rho^{2} \neq q^{-2(r+s-3)}$ by arguments similar to those as above. The only difference is that we have to use conjugates of $\gamma$ instead of bipartition $\gamma$ in the previous arguments. We leave the details to the reader.

Theorem 6.10. Suppose $r, s \in \mathbb{Z}^{>0}$. Then $\mathscr{B}_{r, s}$ is (split) semisimple over $\kappa$ if and only if $e>\max \{r, s\}$ and one of the following conditions holds:

a) $\delta \neq 0$ and $\rho^{2} \neq q^{2 a}$ for any $a \in \mathbb{Z}$ with $|a| \leq r+s-2$.

b) $\delta=0$ and $(r, s) \in\{(1,2),(2,1),(1,3),(3,1)\}$.

Proof. Since $\mathscr{H}_{r} \otimes \mathscr{H}_{s} \cong \mathscr{B}_{r, s} /\left\langle e_{1}\right\rangle$, where $\left\langle e_{1}\right\rangle$ is the two-sided ideal of $\mathscr{B}_{r, s}$ generated by $e_{1}$, $\mathscr{B}_{r, s}$ is not semisimple if $e \leq \max \{r, s\}$. So, we can assume $e>\max \{r, s\}$ when we discuss the semisimplicity of $\mathscr{B}_{r, s}$. We remark that the result for $\delta \neq 0$ follows from Theorem[5.1(b), Lemmas 6.5 6.6 and Proposition 6.9.

Suppose $\delta=0$. By Theorem 5.3, $\mathscr{B}_{r, r}$ is not semisimple for all $r \in \mathbb{Z}^{>0}$. In the remaining part of the proof, we assume $r>s$ since $\mathscr{B}_{r, s} \cong \mathscr{B}_{s, r}$.

When $r+s<5$, either $r=2, s=1$ or $r=3$ and $s=1$. By Lemmas 6.5 6.6, $\operatorname{det} G_{1, \lambda} \neq 0$ for $\lambda \in\left\{((1), \emptyset),((2), \emptyset),\left(\left(1^{2}\right), \emptyset\right)\right\}$. So, both $\mathscr{B}_{2,1}$ and $\mathscr{B}_{3,1}$ is semisimple over $\kappa$. Now, we assume $r+s \geq 5$.

It is easy to see that the Gram matrix $G_{1, \lambda}$ for $\lambda \in\left\{((2),(1)),((2,1), \emptyset),\left(\left(1^{3}\right),(1)\right)\right\}$ is $a \times a$ matrix with $a \in\{6,8\}$. We use MATLAB software to check $\operatorname{det} G_{1, \lambda}=0$. So, $\mathscr{B}_{r, s}$ is not semisimple if $r+s=5$. Further, $\operatorname{Rad} C(1, \lambda)$ contains a non-zero irreducible module, say $D^{\ell, \mu}$, such that $(\ell, \mu)<(1, \lambda)$. We claim $\ell=0$. Otherwise, Applying the functor $\mathcal{F}$ to both $C(1, \lambda)$ and $C(1, \mu)$ yields a non-zero homomorphism from $C(0, \mu)$ to $C(0, \lambda)$. Since we are assuming $o\left(q^{2}\right)>\max \{r, s\}$, both $C(0, \mu)$ and $C(0, \lambda)$ are irreducible. Therefore, $\lambda=\mu$, 
a contradiction. So, $\ell=0$. Applying the functor $G$ to both $C(0, \mu)$ and $C(1, \lambda)$, repeatedly yields a non-zero homomorphism from $C(k, \mu)$ to $C(1+k, \lambda)$ with $2 k+|\mu|=r+s$. Since $(k, \mu)<(1+k, \lambda), \operatorname{det} G_{1+k, \lambda}=0$. By Theorem 5.1(b), $\mathscr{B}_{r, s}$ is not semisimple if $r+s \geq 5$ and $r=s+\ell$ with $\ell \in\{1,2,3\}$.

Finally, we assume $r=s+(k+2)$ with $k \geq 2$ and $r+s>5$. We claim $\operatorname{det} G_{1, \lambda}=0$ if $\lambda=\left(\left(2,1^{k}\right), \emptyset\right)$ for $k \geq 1$. If so, standard arguments on the functor $\mathcal{G}$ shows that $\operatorname{det} G_{s, \lambda}=0$ and hence, by Theorem $5.1(\mathrm{~b}), \mathscr{B}_{r, s}$ is not semisimple.

Suppose $\operatorname{det} G_{1, \lambda} \neq 0$. Then $C(1, \lambda)=D^{1, \lambda}$. We have already verified $\operatorname{det} G_{1,((2,1), \emptyset)}=0$. In general, by Theorem 4.15, $C(1, \mu)$ is a submodule of $\operatorname{Res}^{L} C(1, \lambda)$ where $\mu=\left(\left(2,1^{k-1}\right), \emptyset\right)$. By induction assumption, we have $\operatorname{det} G_{1, \mu}=0$. So, $\operatorname{Rad} C(1, \mu)$ contains an irreducible $\mathscr{B}_{r-1, s}$-module $D^{f, \nu}$ with $(f, \nu)<(1, \mu)$. We have $f \neq 1$. Otherwise, applying the functor $\mathcal{F}$ to both $C(1, \mu)$ and $C(1, \nu)$ yields $\nu=\mu$, a contradiction. So, $f=0$. Since we are assuming $\rho^{2}=1$, by Lemma 6.4, $\nu=\left(\left(2^{2}, 1^{k-2}\right),(1)\right)$. By Frobenius reciprocity, $\operatorname{Hom}\left(\operatorname{Ind}^{L} C(0, \nu), C(1, \lambda) \neq 0\right.$. Using Proposition 4.7 yields $\operatorname{Ind}^{L} C(0, \nu)=\operatorname{Res}^{R} C(1, \nu)$. By Theorem 4.15, this module has a filtration of cell modules such that each section is of form either $C\left(1,\left(\left(2^{2}, 1^{k-2}\right), \emptyset\right)\right)$ or $C(0, \gamma)$ 's for some $\gamma$, where each $\gamma$ can be obtained from $\left(2^{2}, 1^{k-2}\right)$ by adding an addable node. So, $C(1, \lambda)=D^{1, \lambda}$ has to be a composition factor of either $C\left(1,\left(\left(2^{2}, 1^{k-2}\right), \emptyset\right)\right)$ or $C(0, \gamma)$, forcing $\lambda=\left(\left(2^{2}, 1^{k-2}\right), \emptyset\right)$, a contradiction. So, our claim follows.

\section{REFERENCES}

[1] A. Cox, M. De Visscher, S. Doty, And P. Martin, "On the blocks of walled Brauer algebras ", J. Algebra 320, (2008), 169-212.

[2] E. Cline, B. Parshall and L. Scott, "Finite-dimensional algebras and highest weight categories",J. Reine Angew. Math., 391 (1988), 85-99.

[3] R. Dipper, S. Doty, F. Stoll , "The quantized walled Brauer algebra and mixed tensor space", preprint, (2009).

[4] R. Dipper, S. Doty, F. Stoll , "Quantized mixed tensor space and Schur-Weyl duality", preprint, (2009).

[5] R. Dipper and G. James, "Representations of Hecke algebras of finite general linear groups", Proc. London Math. Soc. 52(3), 20-52 (1986).

[6] W. Doran, D. Wales, and P. Hanlon, "On the semisimplicity of the Brauer centralizer algebras", J. Algebra 211 (1999) 647-685.

[7] J. ENYAnG, "Specht modules and semisimplicity criteria for Brauer and Birman-Murakami-Wenzl algebras", J. Algebra Comb. 26 (2007), 291-341.

[8] J. ENYANG, "Cellular bases of the two-parameter version of the centraliser algebra for the mixed tensor representations of the quantum general linear group", Combinatorial representation theory

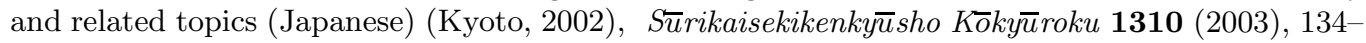
153.

[9] J. Graham and G. Lehrer, "Cellular algebras", Invent.Math. 123 (1996), 1-34.

[10] J. A. Green, "Polynomial representations of $G L_{n}$, 2nd ed., with an appendix on Schensted correspondence and Littlemann paths by K. Erdermann, J. A. Green and M. Schocker", Lecture Notes in Mathematics, 830, Springer-Verlag, Berlin, 2007.

[11] M. Kosuda And J. Murakami, "Centralizer algebras of the mixed tensor representations of quantum group $U_{q}(\operatorname{gl}(n, \mathbf{C})) "$ Osaka J. Math., 30 (1993), 475-507.

[12] R. LEDUC, "A two-parameter version of the centralizer algebra of the mixed tensor representation of the general linear group and quantum general linear group", thesis, University of WisconsinMadison, (1994). 
[13] A. Mathas, "Hecke algebras and Schur algebras of the symmetric group", Univ. Lecture Notes, 15, Amer. Math. Soc., 1999.

[14] G. E. Murphy "The representations of Hecke algebras of type $A_{n}$ " J. Algebra, 173 (1995), $97-121$.

[15] H. Rui And L. Song "The representations of quyantized walled Brauer algebras ", preprint, 2013.

[16] S. Yu "The Cyclotomic BirmanMurakamiWenzl Algebras" , Ph.D thesis, Sydney University (2007)

H.R. Department of Mathematics, East China Normal University, Shanghai, 200062, China E-mail address: hbrui@math.ecnu.edu.cn

L.S. Department of Mathematics, East China Normal University, Shanghai, 200062, China E-mail address: 52110601013@student.ecnu.edu.cn 